\title{
Reconfiguration of Three-Craft Coulomb Formation Based on Patched-Conic-Section Trajectories
}

\author{
Yinan $\mathrm{Xu} *$ \\ University of Michigan, Ann Arbor, Michigan 48109 \\ and \\ Shuquan Wang $\ddagger$ \\ Chinese Academy of Sciences, 100094 Beijing, People's Republic of China \\ DOI: $10.2514 / 1 . G 001557$
}

\begin{abstract}
This paper investigates the nonequilibrium fixed-shape three-craft Coulomb formation reconfiguration problem. Being aware that using the feedback-control approach might result in the chattering of the charges due to the nonequilibrium nature of the system dynamics, this paper proposes a trajectory planning approach to accomplish the reconfiguration. Entire maneuver trajectories are divided into multiple phases. During each phase, only two of the three craft are charged. In this way, the relative trajectory of the charged spacecraft during a certain phase is a conic section. Entire trajectories are composed of patched conics and/or straight lines. The procedures determining the three-phase maneuver strategy are developed, including a preadjusting phase and two transition phases. Numerical simulations demonstrate the effectiveness of the algorithm and the elegance of the charge histories.
\end{abstract}

\section{Introduction}

$\mathbf{T}$ HE concept of Coulomb formation flying (CFF) was introduced by Lyon B. King in 2001 [1]. CFF uses the interspacecraft electrostatic force/forces to control the relative motion of the formation. The electric field is generated onboard by ejecting ions of a certain sign of charge using the ion engine. CFF has the following major advantages:

1) It is power efficient. It requires power level of only $10^{-3} \mathrm{~W}$

2) It is clean. It does not generate the plume impingement, which may cause damages to the nearby spacecraft.

3 ) It is essentially propellantless, which may enhance the life circle of the spacecraft.

On the other side, CFF poses challenges to the electromagnetic compatibility design of the spacecraft. Theoretically, CFF is suitable for a long-period close-proximity space mission in high Earth orbit or deep space.

An important character of CFF is that the Coulomb forces lie only along the line-of-sight directions between spacecraft. Another challenge of CFF is that the space plasma environment shields the strength of the electrostatic force field. This effect will reduce the amount of electrostatic force acting on the neighboring charged spacecraft. The amount of shielding is characterized by the Debye length [2]. At separation distances greater than a Debye length, the intercraft Coulomb forces quickly go to zeros with exponential terms. The Debye length is on the order of centimeters at low Earth orbits, in which the plasma is relatively dense and cold. This results make Coulomb thrusting negligible. However, at geosynchronous Earth orbits, the Debye lengths range between 100 and $1000 \mathrm{~m}[\underline{1}, \underline{3}]$. At one astronomical unit (AU) in deep space, the Debye length ranges around 20-50 m $[1,3]$. This makes the Coulomb thrusting concept feasible for high Earth orbits and deep-space missions when the minimum separation distances are less than $100 \mathrm{~m}$.

Received 2 July 2015; revision received 21 September 2015; accepted for publication 22 September 2015; published online 29 December 2015. Copyright $\odot 2015$ by Shuquan Wang. Published by the American Institute of Aeronautics and Astronautics, Inc., with permission. Copies of this paper may be made for personal or internal use, on condition that the copier pay the $\$ 10.00$ per-copy fee to the Copyright Clearance Center, Inc., 222 Rosewood Drive, Danvers, MA 01923; include the code 1533-3884/15 and $\$ 10.00$ in correspondence with the CCC

*Graduate Student, Mechanical Engineering Department.

${ }^{\dagger}$ Associate Research Fellow, Key Laboratory of Space Utilization, Technology and Engineering Center for Space Utilization; shuquan.wang@ csu.ac.cn.1.
Various mission scenarios of the CFF concept have been investigated. Reference [4] develops a formation feedback-control strategy to achieve the virtual structure control. This control strategy is based on the thrusters' capability to control the three-dimensional motions of the satellites. Schaub and Hussein analyzed the stability of a spinning two-craft Coulomb tether and showed that, if the Debye length was larger than the separation distance, then the nonlinear spinning motion was locally stable; otherwise, the motion was unstable. The perturbed out-of-plane motion was always stable, regardless of Debye length [5]. The nonlinear dynamics and closedloop control are developed for the reconfiguration of a two-satellite Coulomb tether virtual structure near libration points, which is both valid for the robust reconfiguration and the station-keeping mission [6]. Wang and Schaub [7] designed a two-stage charge feedbackcontrol strategy for a one-dimensional constrained Coulomb structure and analyzed the condition for symmetric relative motions of the Coulomb structure to be stabilizable by investigating the total energy of the system. Hussein and Schaub [8] derived the collinear three-craft spinning family of solutions. Feedback control based on the linearized model was designed to stabilize a collinear virtual Coulomb tether system. Asymptotic stability was achieved if the system's angular momentum was equivalent to the estimated/ nominal angular momentum, which was used to calculate the nominal charges [8]. Hogan and Schaub [9] investigated invariant shape conditions for a three-craft collinear formation and proved that, for any collinear formations, there always exists an infinite set of real open-loop charges for equilibrium. Hogan and Schaub [10] also explored the solution families for collinear three-craft $\overline{\mathrm{CFF}}$, and distinct regions where invariant shape solutions exist were analyzed to determine what range of trajectories were possible. Considering the challenges in maintaining and maneuvering inherently unstable formations, Jones and Schaub [11] demonstrated in-plane perturbations could be asymptotically stabilized for the radially aligned configurations. Jasch et al. [12] extended the analysis to the out-of-plane motion and developed a simple control law, which was successful in eliminating a wide variety of out-of-plane perturbations. In [13], an innovative hybrid propulsion strategy was developed by combining Coulomb forces and conventional electric/ion thrusters for closeproximity formation flying. Vasavada and Schaub presented analytical tools to determine the charge solution for a static four-craft formation [14].

This paper focuses on the nonequilibrium reconfiguration problem of the three-craft Coulomb formation. This problem is challenging because controlling all three sides simultaneously is not applicable. Previous research on this problem used a feedback-control approach [15]. Wang and Schaub [15] developed a stable switched control 
strategy based on three Lyapunov-like functions. It achieved stability during the reconfiguration process. But, this approach resulted in the chattering of the charges due to the switched strategy. The chattering may cause a tremendous increase of the power consumption and might even damage the ion engine.

Inspired by [16], which developed patched conic-section trajectories of a two-body Coulomb formation flying, the reconfiguration of the three-craft CFF could be achieved in a much smoother manner if the dynamical properties of the system were fully used. This paper proposes to develop a trajectory programming approach to accomplish the reconfiguration. The entire trajectories are divided into multiple phases. During each phase, only two of the three craft are charged, such that these two craft possess the properties of a two-body problem. In this way, the trajectory of each spacecraft is composed of conic sections and/or straight lines. This is the fundamental idea of this paper. In developing the maneuver trajectories, many specific problems need to be solved, such as the number of phases necessary for the reconfiguration problem, the relationship between the individual spacecraft and the reconfiguration mission, how to choose the two spacecraft to be charged, and so on. These problems are to be answered in the following sections.

\section{Equations of Motion}

The objective of the reconfiguration problem is to make the freeflying three-craft formation formulate the prescribed configuration. The prescribed configuration is defined by the three separation distances: $\left[l_{12}^{*}, l_{23}^{*}, l_{13}^{*}\right]$. Figure 1 shows the scenario of this three-body Coulomb virtual structure.

Assuming that the size of spacecraft is much smaller than the separation distance, the point-charge models are used to model the electric forces. The Coulomb force between $i$ th and $j$ th spacecraft exerted by the $i$ th spacecraft is given by

$$
\boldsymbol{F}_{i j}=k_{c} \frac{q_{i} q_{j}}{r_{i j}^{3}}\left(1+\frac{r_{i j}}{\lambda_{d}}\right) \exp \left(-\frac{r_{i j}}{\lambda_{d}}\right) \boldsymbol{r}_{i j}
$$

where $k_{c}=8.99 \times 10^{9} \mathrm{Nm}^{2} \cdot \mathrm{C}^{-2}$ is the Coulomb constant, $q_{i}$ are the charges of the $i$ th spacecraft that can be actively controlled, $r_{i j}=\left\|r_{i j}\right\|$ is the separation distance between the $i$ th and $j$ th spacecraft, and $\boldsymbol{r}_{i j}$ is the relative position vector pointing from the $i$ th to the $j$ th spacecraft. The parameter $\lambda_{d}$ is the Debye length, which characterizes the strength of the plasma shielding effect. It is influenced by the temperature and the ion/electron density. For high Earth orbits, the Debye length ranges between 100 and $1000 \mathrm{~m}$ $[1,17]$. In deep space at a one-astronomical-unit distance from the sun, the Debye length can vary between 30 and $50 \mathrm{~m}$. CFF typically has spacecraft separation distances less than $100 \mathrm{~m}$. Generally, it is assumed that the Coulomb thrusting is applicable only when the separation distance is less than the local Debye length. In developing the maneuver strategy, it is assumed that $\lambda_{d}=\infty$ such that many analytical results can be used. In this way, Eq. (1) is simplified to be

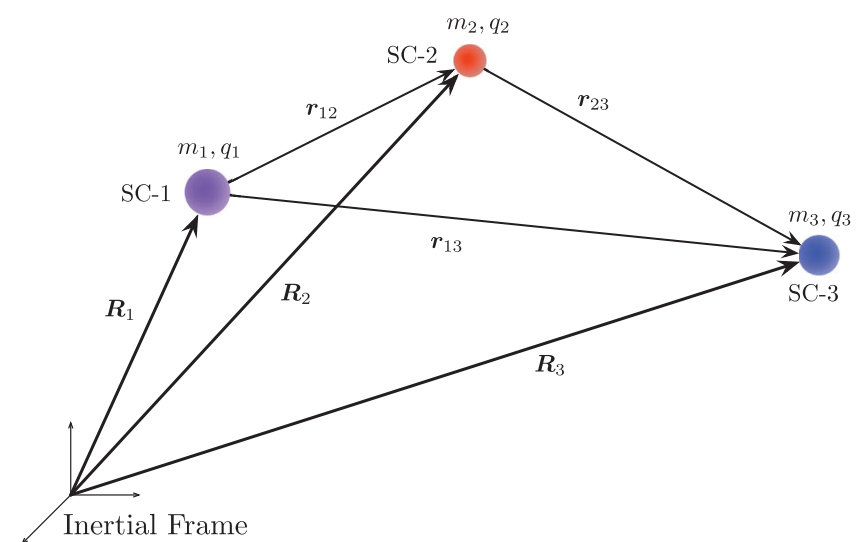

Fig. 1 Scenario of the three-craft Coulomb formation in free space.

$$
\boldsymbol{F}_{i j}=k_{c} \frac{q_{i} q_{j}}{r_{i j}^{3}} \boldsymbol{r}_{i j}
$$

By the assumption that there are no external forces acting on the three-body system, the inertial equations of motion are given by

$$
\begin{gathered}
m_{1} \ddot{\boldsymbol{R}}_{1}=-\frac{k_{c} q_{1} q_{2}}{r_{12}^{3}} \boldsymbol{r}_{12}-\frac{k_{c} q_{1} q_{3}}{r_{13}^{3}} \boldsymbol{r}_{13} \\
m_{2} \ddot{\boldsymbol{R}}_{2}=\frac{k_{c} q_{1} q_{2}}{r_{12}^{3}} \boldsymbol{r}_{12}-\frac{k_{c} q_{2} q_{3}}{r_{23}^{3}} \boldsymbol{r}_{23} \\
m_{3} \ddot{\boldsymbol{R}}_{3}=\frac{k_{c} q_{1} q_{3}}{r_{13}^{3}} \boldsymbol{r}_{13}+\frac{k_{c} q_{2} q_{3}}{r_{23}^{3}} \boldsymbol{r}_{23}
\end{gathered}
$$

where $m_{i}$ is the mass of the $i$ th spacecraft, and $\boldsymbol{R}_{i}$ is the position vector of the $i$ th spacecraft in the inertial frame.

\section{Single-Phase Maneuver Trajectory of Spacecraft-1 and Spacecraft-3 Subsystem}

This section investigates the single-phase trajectory for the reconfiguration mission. The three-craft system is separated into two groups: a subsystem of two charged spacecraft, and an uncharged spacecraft. Without loss of generality, spacecraft (SC)-1 and SC-3 are chosen as the charged spacecraft. The relative trajectory of the SC-1 and SC-3 subsystem is a conic section. SC-2 is stationary or moving in a straight line. Substituting $q_{2}=0$ into Eq. (3), the equations of motions reduce to

$$
\begin{gathered}
m_{1} \ddot{\boldsymbol{R}}_{1}=-\frac{k_{c} q_{1} q_{3}}{r_{13}^{3}} \boldsymbol{r}_{13} \\
m_{2} \ddot{\boldsymbol{R}}_{2}=0 \\
m_{3} \ddot{\boldsymbol{R}}_{3}=\frac{k_{c} q_{1} q_{3}}{r_{13}^{3}} \boldsymbol{r}_{13}
\end{gathered}
$$

It is still complicated to find the connection between the motions of the individual spacecraft and the desired triangular shape. Note that there are no external forces acting on this system, the center of mass (CM) of the SC-1 and SC-3 subsystem has a simple type of motion. The investigation initiates by studying the motion of the $\mathrm{CM}$ of the subsystem. Figure $\underline{2}$ shows the scenario of this case as seen from

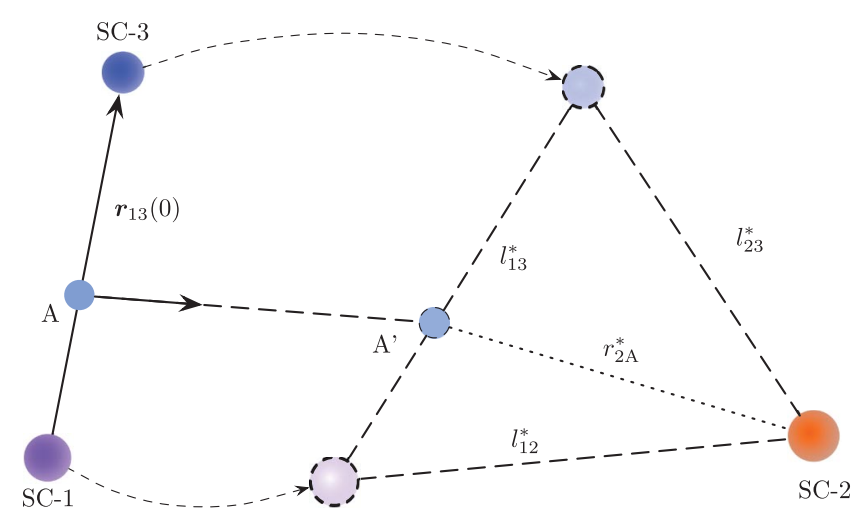

Fig. 2 CM motion of the SC-1 and SC-3 subsystem viewed from SC-2. 
$\mathrm{SC}-2$. The $\mathrm{CM}$ of the subsystem is denoted by point $\mathrm{A}$, and the final position of the CM is denoted by A'.

\section{A. Subsystem's Center of Mass Motion Requirements}

Because point $\mathrm{A}$ is the $\mathrm{CM}$ of the $\mathrm{SC}-1$ and $\mathrm{SC}-3$ subsystem, the states of point A satisfy

$$
\begin{gathered}
\boldsymbol{R}_{\mathrm{A}}=\frac{m_{1} \boldsymbol{R}_{1}+m_{3} \boldsymbol{R}_{3}}{m_{1}+m_{3}} \\
\dot{\boldsymbol{R}}_{\mathrm{A}}=\frac{m_{1} \dot{\boldsymbol{R}}_{1}+m_{3} \dot{\boldsymbol{R}}_{3}}{m_{1}+m_{3}}
\end{gathered}
$$

Equations (ㅁ) and (ㅁ) are valid at any time during this phase. The relative position vector pointing from SC-1 to $\mathrm{SC}-3$ is given by

$$
\boldsymbol{r}_{13}=\boldsymbol{R}_{3}-\boldsymbol{R}_{1}
$$

Using Eqs. (ㄷ) and (7), $\boldsymbol{R}_{1}$ and $\boldsymbol{R}_{3}$ are expressed in terms of $\boldsymbol{R}_{\mathrm{A}}$ and $\boldsymbol{r}_{13}$ as

$$
\begin{aligned}
& \boldsymbol{R}_{1}=\boldsymbol{R}_{\mathrm{A}}-\frac{m_{1}}{m_{1}+m_{3}} \boldsymbol{r}_{13} \\
& \boldsymbol{R}_{3}=\boldsymbol{R}_{\mathrm{A}}+\frac{m_{3}}{m_{1}+m_{3}} \boldsymbol{r}_{13}
\end{aligned}
$$

Because there are no external forces acting on the SC- 1 and SC-3 subsystem, $\dot{\boldsymbol{R}}_{\mathrm{A}}$ remains constant. The location of point A at time $t$ is given by

$$
\boldsymbol{R}_{\mathrm{A}}(t)=\boldsymbol{R}_{\mathrm{A}}(0)+\dot{\boldsymbol{R}}_{\mathrm{A}}(0) \cdot t
$$

where $\boldsymbol{R}_{\mathrm{A}}(0)$ and $\dot{\boldsymbol{R}}_{\mathrm{A}}(0)$ are the initial position and velocity vector of point A. Similarly, the position vector of SC-2 in the inertial frame is given by

$$
\boldsymbol{R}_{2}(t)=\boldsymbol{R}_{2}(0)+\dot{\boldsymbol{R}}_{2}(0) \cdot t
$$

Now, the connection between the motion of point $A$ and the desired triangular configuration is ready to be investigated. Figure 3 illustrates the desired triangle. Note that, for the desired triangle, the three side lengths $\left[l_{12}^{*}, l_{23}^{*}, l_{13}^{*}\right]$ are preset. Point A lies on the line segment $S_{1} S_{3}$. The distance from SC-1 to point A is

$$
r_{1 \mathrm{~A}}^{*}=\frac{m_{3}}{m_{1}+m_{3}} l_{13}^{*}
$$

Applying the law of cosines to the triangle $\Delta S_{1} A S_{2}$, the distance between point $\mathrm{A}$ and SC-2 is obtained:

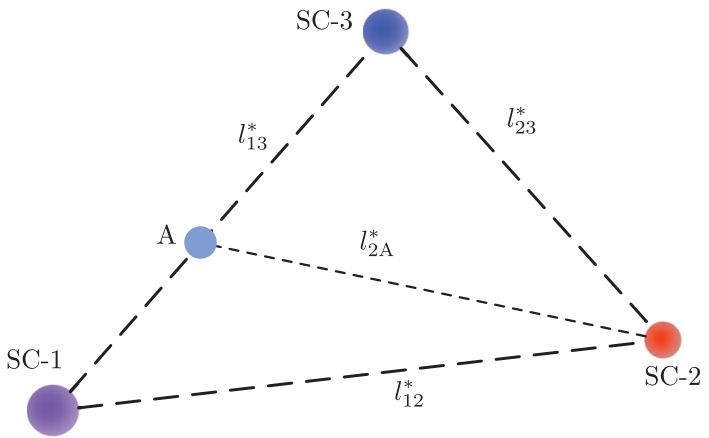

Fig. 3 Desired triangle.

$$
r_{2 \mathrm{~A}}^{*}=\sqrt{r_{1 \mathrm{~A}}^{* 2}+l_{12}^{* 2}-2 r_{1 \mathrm{~A}}^{*} l_{12}^{*} \cos \angle S_{1}^{*}}
$$

where $\cos \angle S_{1}$ is calculated by

$$
\cos \angle S_{1}=\frac{l_{12}^{* 2}+l_{13}^{* 2}-l_{23}^{* 2}}{2 l_{12}^{*} l_{13}^{*}}
$$

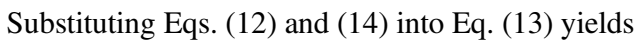

$$
r_{2 \mathrm{~A}}^{*}=\frac{1}{m_{1}+m_{3}} \sqrt{m_{1}\left(m_{1}+m_{3}\right) l_{12}^{2}-m_{1} m_{3} l_{13}^{2}+m_{3}\left(m_{1}+m_{3}\right) l_{23}^{2}}
$$

In Eq. (15), $r_{2 A}^{*}$ is expressed in terms of the three side lengths of the triangle and the masses of the three craft. Equation (15) is a property of the desired triangular formation. This is a necessary condition for the existence of the solution to the reconfiguration problem to rephrase. The necessary condition is that there exists a time $t>0$ such that

$$
r_{2 \mathrm{~A}}(t)=r_{2 \mathrm{~A}}^{*}
$$

is satisfied.

The necessary condition in Eq. (16) is a connection between the desired triangle and the relative motion of point A and SC-2. Note that $r_{2 \mathrm{~A}}$ is the magnitude of the relative position vector pointing from SC2 to point $\mathrm{A}$ :

$$
r_{2 \mathrm{~A}}(t)=\left\|\boldsymbol{r}_{2 \mathrm{~A}}(t)\right\|=\left\|\boldsymbol{R}_{\mathrm{A}}(t)-\boldsymbol{R}_{2}(t)\right\|
$$

Substituting Eq. (17) into Eq. (16) yields

$$
\left\|\boldsymbol{R}_{\mathrm{A}}(t)-\boldsymbol{R}_{2}(t)\right\|=r_{2 \mathrm{~A}}^{*}
$$

Squaring both sides in Eq. (18) yields

$$
\boldsymbol{R}_{\mathrm{A}}^{2}(t)+\boldsymbol{R}_{2}^{2}(t)-2 \boldsymbol{R}_{\mathrm{A}}(t) \cdot \boldsymbol{R}_{2}(t)=r_{2 \mathrm{~A}}^{* 2}
$$

Substitute Eqs. (10) and (11) into Eq. (19), Eq. (19) can then be expressed in the scalar form

$$
v_{2 \mathrm{~A}}^{2}(0) t^{2}+2 r_{2 \mathrm{~A}}(0) v_{2 \mathrm{~A}}(0) \cos \theta_{0} t+r_{2 \mathrm{~A}}^{2}(0)-r_{2 \mathrm{~A}}^{* 2}=0
$$

where $v_{2 \mathrm{~A}}=\left\|\dot{\boldsymbol{r}}_{2 \mathrm{~A}}\right\|$, and $\theta \in[0, \pi]$ is the angle between $\boldsymbol{r}_{2 \mathrm{~A}}$ and $\dot{\boldsymbol{r}}_{2 \mathrm{~A}}$. The necessary condition in Eq. (16) is transformed to be the existence of at least one positive solution to the quadratic equation about time $t$ in Eq. (20). The coefficients of this quadratic equation are determined by the initial states, the masses, and the required three side lengths of the three-craft system.

Generally, there are two solutions to Eq. (20):

$$
t_{1}=\frac{-r_{2 \mathrm{~A}}(0) \cos \theta_{0}+\sqrt{r_{2 \mathrm{~A}}^{* 2}-r_{2 \mathrm{~A}}^{2}(0) \sin ^{2} \theta_{0}}}{v_{2 \mathrm{~A}}(0)}
$$

$$
t_{2}=\frac{-r_{2 \mathrm{~A}}(0) \cos \theta_{0}-\sqrt{r_{2 \mathrm{~A}}^{* 2}-r_{2 \mathrm{~A}}^{2}(0) \sin ^{2} \theta_{0}}}{v_{2 \mathrm{~A}}(0)}
$$

Since $t_{1} \geq t_{2}$, for Eq. (20) to have at least one positive solution, both of the following inequalities must be satisfied:

$$
r_{2 \mathrm{~A}}^{* 2}-r_{2 \mathrm{~A}}^{2}(0) \sin ^{2} \theta_{0} \geq 0
$$

$$
\sqrt{r_{2 \mathrm{~A}}^{* 2}-r_{2 \mathrm{~A}}^{2}(0) \sin ^{2} \theta_{0}}>r_{2 \mathrm{~A}}(0) \cos \theta_{0}
$$


Table 1 Cases of solutions to Eq. (20)

\begin{tabular}{|c|c|c|c|}
\hline Equation (23a) & Equation (24) & Equation (25) & Solutions to Eq. (20) \\
\hline \multirow[t]{4}{*}{ True } & \multirow[t]{2}{*}{ True } & False & Two positive solutions \\
\hline & & True & One positive solution \\
\hline & \multirow[t]{2}{*}{ False } & True & One positive solution \\
\hline & & False & Two negative solutions \\
\hline \multirow[t]{4}{*}{ False } & \multirow[t]{2}{*}{ True } & True & $\mathrm{N} / \mathrm{A}^{\mathrm{a}}$ \\
\hline & & False & $\begin{array}{l}\text { Imaginary solutions with } \\
\text { positive real part }\end{array}$ \\
\hline & \multirow[t]{2}{*}{ False } & True & $\mathrm{N} / \mathrm{A}^{\mathrm{a}}$ \\
\hline & & False & $\begin{array}{l}\text { Imaginary solutions with } \\
\text { negative real part }\end{array}$ \\
\hline
\end{tabular}

aN/A denotes "not applicable."

The inequality in Eq. (23a) ensures that the solutions to Eq. (20) are real. Equation $(23 \mathrm{~b})$ guarantees that $t_{1}>0$. Note that, if

$$
\frac{\pi}{2}<\theta_{0}<\pi
$$

then $\cos \theta_{0}<0$; thus, Eq. (23b) is always true if Eq. (23a) can be guaranteed. Otherwise, for $\mathrm{Eq}$. (23b) to be true, the following inequality must be satisfied:

$$
r_{2 \mathrm{~A}}<r_{2 \mathrm{~A}}^{*}
$$

Moreover, if the following inequality is also satisfied,

$$
\sqrt{r_{2 \mathrm{~A}}^{* 2}-r_{2 \mathrm{~A}}^{2}(0) \sin ^{2} \theta_{0}}<-r_{2 \mathrm{~A}}(0) \cos \theta_{0}
$$

then $t_{1}$ and $t_{2}$ are both positive. If

$$
0<\theta_{0}<\frac{\pi}{2}
$$

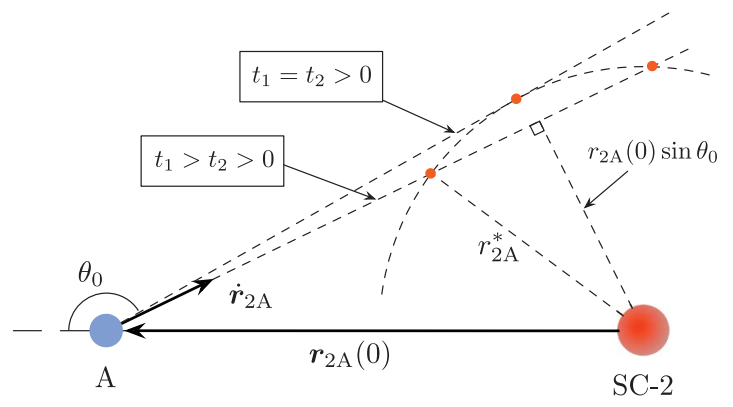

a) Two positive solutions

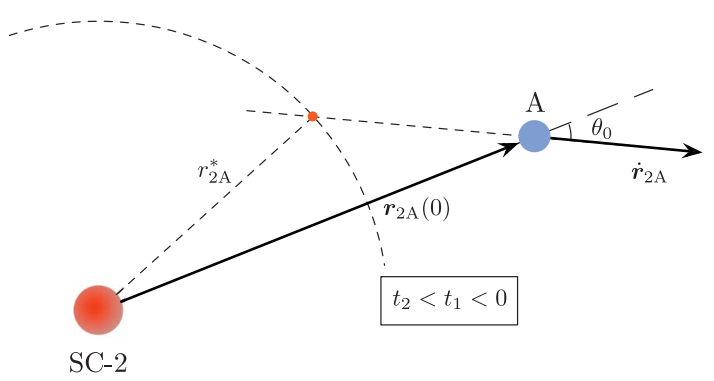

c) Two real solutions, but no positive solutions then Eq. (26) cannot be satisfied. For Eq. (드) to be true, the following inequalities must be satisfied:

$$
\begin{aligned}
& \frac{\pi}{2}<\theta_{0}<\pi \\
& r_{2 \mathrm{~A}}>r_{2 \mathrm{~A}}^{*}
\end{aligned}
$$

Table 1 summarizes the cases of the solutions to Eq. (20). Figure 4 illustrates the scenarios of the typical cases. Note that the scenarios shown in Fig. 4 are the relative motion of point A as seen from SC-2. Figure 4a shows the case where both of the three inequalities in Eqs. (23a), (23b), and (26) are satisfied. As shown in Fig. 4b, point A moves along the dashed straight line defined by the velocity $\dot{\boldsymbol{r}}_{2 \mathrm{~A}}$ and the initial positive vector $\boldsymbol{r}_{2 \mathrm{~A}}(0)$. Equation (23a) ensures that the distance between SC-2 and the dashed straight line is not greater than $r_{2 \mathrm{~A}}^{*}$. Thus, the dashed straight line either has two cross points or has one tangential point with the sphere. In both cases, Eq. (20) has two real solutions: distinct or identical, as shown in Fig. 4a. Equations (23b) and (26) ensure that the two solutions are positive.

Figure $4 \mathrm{~b}$ shows the case where the two inequalities in Eqs. (23a) and $(23 b)$ are satisfied, which means that Eq. (20) has one positive and one negative solution. It can be seen that, in this case, the initial position of point $\mathrm{A} \boldsymbol{r}_{2 \mathrm{~A}}(0)$ is within the sphere defined by $r_{2 \mathrm{~A}}^{*}$. Figure $4 \mathrm{c}$ illustrates the case where the two solutions to Eq. (20) are both negative. In this case, only Eq. (23a) is satisfied. Geometrically, point $\mathrm{A}$ is moving in the direction that is opposite to the sphere defined by $r_{2 \mathrm{~A}}^{*}$.

Satisfying the inequalities in Eqs. (23a) and (23b) ensures that the distance between point A and SC-2 will reach the desired value. The desired value of the distance is calculated according to the expected triangular configuration. Note that the inequalities in Eqs. (23a) and (23b) can be verified based on the initial states of the three-craft system and the desired triangular configuration. The inequalities in

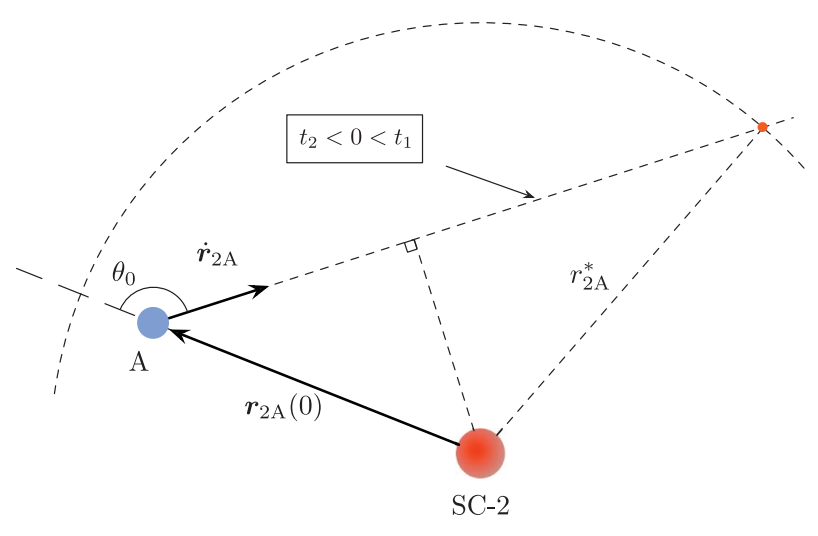

b) One positive solution

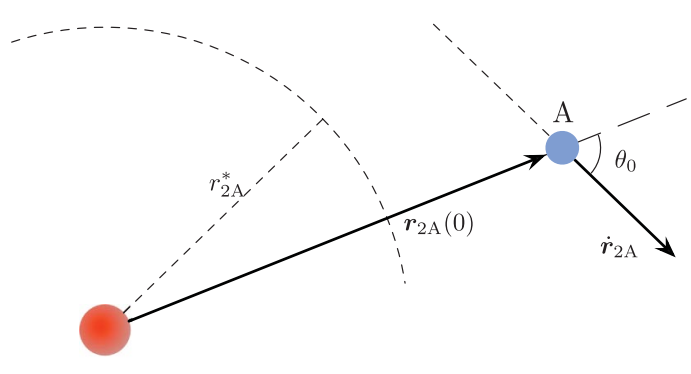

SC-2

d) No real solutions

Fig. 4 Cases of solutions to Eq. (20). 
Eqs. (23a) and (23b) are necessary for the existence of a one-phase trajectory that accomplishes the reconfiguration task. The next item to be investigated is the relative motion of the SC-1 and SC-3 subsystem.

\section{B. Spacecraft-1 and Spacecraft-3 Relative Motion}

Assuming that $t^{*}$ is a positive solution to Eq. (20), this section investigates the motions of SC-1 and SC-3. The objective is to find a proper relative motion of SC-1 and SC-3 such that the three-craft system reaches the desired configuration at time $t^{*}$. Specifically, given the initial states of the system and the maneuver time $t^{*}$, find the charge product $Q_{13} \triangleq q_{1} q_{3}$ such that the following equations are satisfied:

$$
\left[l_{12}(t), l_{23}(t), l_{13}(t)\right]=\left[l_{12}^{*}, l_{23}^{*}, l_{13}^{*}\right]
$$

$$
t=t^{*}
$$

First, let us explore the connection between the geometry of the desired triangle and the corresponding states of the system. According to the maneuver time $t^{*}$, the expected states of SC-2 and point $\mathrm{A}$ are given by

$$
\begin{aligned}
& \boldsymbol{R}_{2}^{*}=\boldsymbol{R}_{2}(0)+\dot{\boldsymbol{R}}_{2} \cdot t^{*} \\
& \boldsymbol{R}_{\mathrm{A}}^{*}=\boldsymbol{R}_{\mathrm{A}}(0)+\dot{\boldsymbol{R}}_{\mathrm{A}} \cdot t^{*}
\end{aligned}
$$

Note that there are no external forces acting on the SC-1 and SC-3 subsystem. The relative motion of SC-1 and SC-3 is planar due to the conservation of the angular momentum. The normal direction of the orbit plane is defined by the specific angular momentum of the subsystem:

$$
\boldsymbol{h}_{13}=\boldsymbol{r}_{13}(0) \times \dot{\boldsymbol{r}}_{13}(0)
$$

At time $t^{*}$, the relative position vector pointing from $\mathrm{SC}-1$ to point A $\boldsymbol{r}_{1 \mathrm{~A}}$ must satisfy

$$
\boldsymbol{r}_{1 \mathrm{~A}}\left(t^{*}\right) \cdot \boldsymbol{h}_{13}=0
$$

$$
\left\|\boldsymbol{r}_{1 \mathrm{~A}}\left(t^{*}\right)\right\|=r_{1 \mathrm{~A}}^{*}
$$

where $r_{1 \mathrm{~A}}^{*}$ is calculated by Eq. (12). Then, $\boldsymbol{r}_{12}(t)$ must satisfy the length requirement:

$$
\left\|\boldsymbol{r}_{12}\left(t^{*}\right)\right\|=l_{12}^{*}
$$

The three equations in Eqs. (35-37) are the equality constraints for the vector $\boldsymbol{R}_{1}^{*}$, which is the expected position of SC- 1 at time $t^{*}$. There are three unknown parameters in the vector $\boldsymbol{R}_{1}^{*}$. Thus, $\boldsymbol{R}_{1}^{*}$ is solved through Eqs. (35-37). Once $\boldsymbol{R}_{1}^{*}$ is obtained, the location of SC-3 is calculated by

$$
\boldsymbol{R}_{3}^{*}=\boldsymbol{R}_{\mathrm{A}}^{*}+\frac{m_{3}}{m_{1}}\left(\boldsymbol{R}_{\mathrm{A}}^{*}-\boldsymbol{R}_{1}^{*}\right)
$$

Note that Eqs. (36) and (37) are essentially quadratic equations. Generally, the three equality constraints in Eqs. (35-37) result in two solutions of $\boldsymbol{R}_{1}^{*}$. This can be explained by the two plots in Fig. 5. In Fig. 5 a, the line connecting SC-1 and SC-3 rotates at an acute angle and then reaches the desired configuration at time $t^{*}$. In Fig. 5b, the same line swipes an obtuse angle until it reaches the desired configuration. In both cases, the three-craft formation reaches the desired configuration at time $t^{*}$. The locations of SC- 1 and SC- 3 are different in the two plots. Flipping over the final triangle in Fig. $\underline{5 a}$ along the line $r_{2 \mathrm{~A}}^{*}$ yields the final triangle in Fig. $5 \mathrm{~b}$.

Using the geometrical properties of the desired triangle, the corresponding relative position vector $\boldsymbol{r}_{13}^{*}$ is found through Eqs. (3538). Once $\boldsymbol{r}_{13}^{*}$ is obtained, the maneuver problem is formulated as giving the initial states $\boldsymbol{r}_{13}(0)$ and $\dot{\boldsymbol{r}}_{13}(0)$, finding the charge product $Q_{13}$ such that the relative position vector $\boldsymbol{r}_{13}$ reaches the desired value $\boldsymbol{r}_{13}^{*}$ at time $t^{*}$. At first glance, this problem seems similar to the Lambert problem. But, the differences between this problem and the Lambert problem are significant and result in a different solution path. The following deductions aim to find the effective gravitational coefficient $\mu^{*}$ such that the expected relative position vector $\boldsymbol{r}_{13}^{*}$ can be reached.

The expected position vector $\boldsymbol{r}_{13}^{*}$ is expressed by the initial states $\left[\boldsymbol{r}_{13}(0), \dot{\boldsymbol{r}}_{13}(0)\right]$ as

$$
\boldsymbol{r}_{13}^{*}=F^{*} \boldsymbol{r}_{13}(0)+G^{*} \dot{\boldsymbol{r}}_{13}(0)
$$

where $F^{*}$ and $G^{*}$ are the Lagrange coefficients. Cross-multiplying both sides of Eq. (푸) with $\dot{\boldsymbol{r}}_{13}(0)$ yields

$$
F^{*} \boldsymbol{r}_{13}(0) \times \dot{\boldsymbol{r}}_{13}(0)=\boldsymbol{r}_{13}^{*} \times \dot{\boldsymbol{r}}_{13}(0)
$$

Note that $\boldsymbol{r}_{13}(0) \times \dot{\boldsymbol{r}}_{13}(0) \triangleq \boldsymbol{h}_{13}$ is the specific angular momentum of the subsystem, which is constant by the assumption that there are no external forces acting on the subsystem. By dot multiplying both sides in Eq. (40) by $\boldsymbol{h}_{13}, F^{*}$ is obtained:
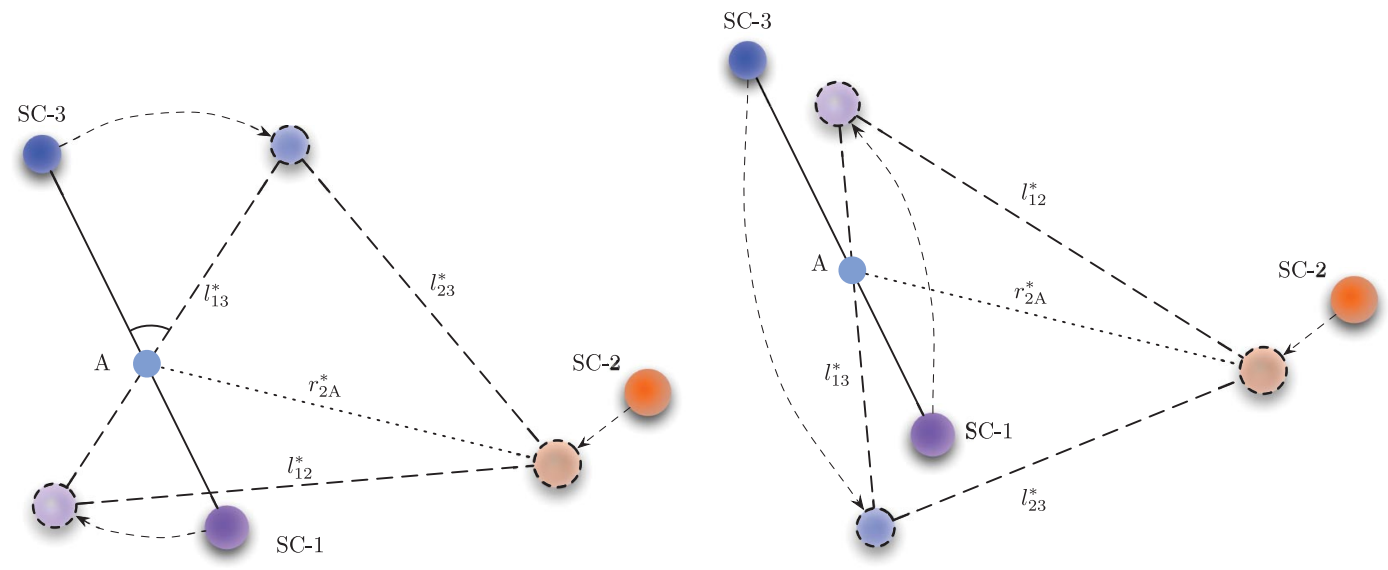

a) The SC-1 SC-3 subsystem rotates an acute angle

b) The SC-1 SC-3 subsystem rotates an obtuse angle

Fig. 5 Illustration of the expected maneuver scenarios. 


$$
F^{*}=\frac{\boldsymbol{r}_{13}^{*} \times \dot{\boldsymbol{r}}_{13}(0) \cdot \boldsymbol{h}_{13}}{h_{13}^{2}}
$$

The Lagrange coefficient $F^{*}$ coefficient can be expressed in terms of the effective gravitational coefficient as

$$
F^{*}=1-\frac{\mu^{*}(1-\cos \gamma)}{h_{13}^{2}} r_{13}^{*}
$$

where $\gamma$ is the angle between the vectors $\boldsymbol{r}_{13}(0)$ and $\boldsymbol{r}_{13}^{*}$. Using Eqs. (41) and (42), the effective gravitational coefficient is obtained:

$$
\mu^{*}=\frac{\left(1-F^{*}\right) h_{13}^{2}}{(1-\cos \gamma) r_{13}^{*}}=\frac{h_{13}^{2}-\boldsymbol{r}_{13}^{*} \times \dot{\boldsymbol{r}}_{13}(0) \cdot \boldsymbol{h}_{13}}{(1-\cos \gamma) r_{13}^{*}}
$$

Logically, given the initial states $\left[\boldsymbol{r}_{13}(0), \dot{\boldsymbol{r}}_{13}(0)\right]$ and the effective gravitational coefficient, the relative trajectory is determined. The time of flight when $\boldsymbol{r}_{13}^{*}$ is reached is also determined. The reciprocal of the semimajor axis is

$$
\alpha=\frac{1}{a}=\frac{2}{r_{13}(0)}-\frac{v_{13}^{2}(0)}{\mu^{*}}
$$

where $r_{13}(0)$ and $v_{13}(0)$ are the magnitude of position vector $\boldsymbol{r}_{13}(0)$ and velocity vector $\dot{\boldsymbol{r}}_{13}(0)$, respectively. The eccentric vector is

$$
\boldsymbol{c}=\dot{\boldsymbol{r}}_{13}(0) \times \boldsymbol{h}_{13}-\mu^{*} \frac{\boldsymbol{r}_{13}(0)}{r_{13}(0)}
$$

The eccentricity is given by

$$
e=\frac{\|c\|}{\left|\mu^{*}\right|}
$$

The initial true anomaly of the subsystem is

$$
f_{13}(0)=\arctan \left(\frac{\left(\hat{\boldsymbol{i}}_{c} \times \hat{\boldsymbol{i}}_{r_{13}(0)}\right) \cdot \hat{\boldsymbol{i}}_{h}}{\hat{\boldsymbol{i}}_{c} \cdot \hat{\boldsymbol{i}}_{r_{13}(0)}}\right)
$$

The true anomaly of $\boldsymbol{r}_{13}^{*}$ is given by

$$
f_{13}^{*}=f_{13}(0)+\gamma
$$

Depending on the values of $\mu^{*}$ and $a$, the possible type of the relative trajectory of the subsystem includes ellipse, parabola, attractive hyperbola, and repulsive hyperbola. Table 2 illustrates the calculation procedures of the time of flight in various cases. It is verified that, once the initial states $\left[\boldsymbol{r}_{13}(0), \dot{\boldsymbol{r}}_{13}(0)\right]$ and the effective gravitational coefficient $\mu^{*}$ are determined, the time of flight $\Delta t$ is also determined.

Note that there are two requirements to the one-phase trajectory. First, the relative position vector will reach the desired value, which is formulated as $\boldsymbol{r}_{13}(\Delta t)=\boldsymbol{r}_{13}^{*}$. Second, the time of flight $\Delta t$ is equal to $t^{*}$. The first requirement is ensured by the value of the effective gravitational coefficient $\mu^{*}$. Once $\mu^{*}$ is obtained, $\Delta t$ is determined. The second requirement is not ensured. Only at certain coincidental occasions will the time requirement be satisfied. Generally, using a one-phase relative trajectory is not enough to accomplish the reconfiguration problem. A more delicate strategy is expected to solve the problem.

\section{Two-Phase Maneuver Trajectory}

\begin{tabular}{|c|c|c|c|}
\hline$\underline{\mu^{*}}$ & $a$ & $\begin{array}{c}\text { Trajectory } \\
\text { type }\end{array}$ & Calculation procedure \\
\hline$\mu^{*}>0$ & $a>0$ & Ellipse & $\begin{array}{l}E_{13}(0)=2 \arctan \left(\sqrt{\frac{1-e}{1+e}}\right) \cdot \tan \frac{f_{13}(0)}{2} \\
E_{13}(\Delta t)=2 \arctan \left(\sqrt{\frac{1-e}{1+e}}\right) \cdot \tan \frac{f_{13}(\Delta t)}{2} \\
M_{13}(0)=E_{13}(0)-e \sin E_{13}(0) \\
M_{13}(\Delta t)=E_{13}(\Delta t)-e \sin E_{13}(\Delta t) \\
\Delta t=\frac{M_{13}(\Delta t)-M_{13}(0)}{\sqrt{\mu^{*} / a^{3}}}\end{array}$ \\
\hline & $a<0$ & $\begin{array}{l}\text { Attractive } \\
\text { hyperbola }\end{array}$ & $\begin{array}{l}H_{13}(0)=2 \operatorname{arctanh}\left(\sqrt{\frac{e-1}{e+1}}\right) \cdot \tan \frac{f_{13}(0)}{2} \\
H_{13}(\Delta t)=2 \operatorname{arctanh}\left(\sqrt{\frac{e-1}{e+1}}\right) \cdot \tan \frac{f_{13}(\Delta t)}{2} \\
N_{13}(0)=e \sinh H_{13}(0)-H_{13}(0) \\
N_{13}(\Delta t)=e \sinh H_{13}(\Delta t)-H_{13}(\Delta t) \\
\Delta t=\frac{N_{13}(\Delta t)-N_{13}(0)}{\sqrt{-\mu^{*} / a^{3}}}\end{array}$ \\
\hline$\mu^{*}<0$ & $a>0$ & $\begin{array}{l}\text { Repulsive } \\
\text { hyperbola }\end{array}$ & $\begin{array}{l}H_{13}(0)=2 \operatorname{arctanh}\left(\sqrt{\frac{e-1}{e+1}}\right) \cdot \tan \frac{f_{13}(0)}{2} \\
H_{13}(\Delta t)=2 \operatorname{arctanh}\left(\sqrt{\frac{e-1}{e+1}}\right) \cdot \tan \frac{f_{13}(\Delta t)}{2} \\
N_{13}(0)=e \sinh H_{13}(0)+H_{13}(0) \\
N_{13}(\Delta t)=e \sinh H_{13}(\Delta t)+H_{13}(\Delta t) \\
\Delta t=\frac{N_{13}(\Delta t)-N_{13}(0)}{\sqrt{-\mu^{*} / a^{3}}}\end{array}$ \\
\hline
\end{tabular}

The difficulty of the one-phase trajectory is that the trajectory has inadequate flexibilities to satisfy both requirements. This section proposes an approach to increase the degrees of freedom of the trajectory design. The relative motion of the SC-1 and SC-3 subsystem is divided into two phases. In this way, two more degrees of freedom are introduced into the trajectory design.
Table 2 Calculations of $\Delta t$ in different situations

Figure 6 illustrates the two-phase trajectory of the SC-1 and SC-3 subsystem. For notational convenience, $\widehat{B C}$ and $\widehat{C D}$ represent the phase 1 and phase 2 trajectories, respectively. All of the trajectories are determined by the charge product of phase $1 Q_{13,1}$, the phase 1 time duration $\Delta t_{1}$, the charge product of phase $2 Q_{13,2}$, and the phase 2 time duration $\Delta t_{2}$. Note that, once $Q_{13,1}$ and $\Delta t_{1}$ are determined, the states of point $\mathrm{C}$ are determined. Thus, the phase 2 trajectory is obtained using the procedure developed in the section about the onephase maneuver trajectory (Sec. III). So, the independent variables in the two-phase maneuver trajectory design problem are $Q_{13,1}$ and $\Delta t_{1}$. The objective of the two-phase trajectory design is to determine the variables $Q_{13,1}$ and $\Delta t_{1}$ such that

$$
\boldsymbol{r}_{13}(t)=\boldsymbol{r}_{13}^{*}
$$

$$
t=t^{*}
$$

are satisfied. Here, $t \triangleq \Delta t_{1}+\Delta t_{2}$, and $t^{*}$ is a positive solution to Eq. (20). The last section shows that, by providing the states of point

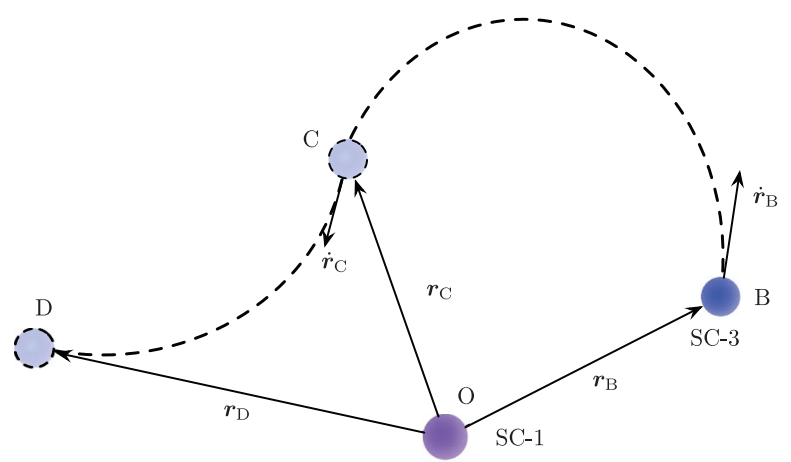

Fig. 6 Two-phase relative trajectory of the SC-1 and SC-3 subsystem. 
C, a trajectory that satisfies Eq. (49) can be found. Essentially, we need to determine two variables such that Eq. (50) is satisfied. Logically, there exists one degree of freedom in this problem.

This section assumes that the charge product $Q_{13,1}$ is given, and then it develops a procedure to determine $\Delta t_{1}$ such that both of the requirements in Eqs. (49) and (50) are satisfied. Because $Q_{13,1}$ is known, the shape of phase 1 is determined. The effective gravitational coefficient of the subsystem in phase 1 is

$$
\mu_{13,1}=-k_{c} Q_{13,1}\left(\frac{1}{m_{1}}+\frac{1}{m_{3}}\right)
$$

The basic orbital characters are obtained as follows:

$$
\begin{gathered}
\alpha_{13,1}=\frac{1}{a_{13,1}}=\frac{2}{r_{\mathrm{B}}}-\frac{v_{\mathrm{B}}^{2}}{\mu_{13,1}} \\
\boldsymbol{c}_{13,1}=\dot{\boldsymbol{r}}_{\mathrm{B}} \times \boldsymbol{h}_{13}-\mu_{13,1} \frac{\boldsymbol{r}_{\mathrm{B}}}{r_{\mathrm{B}}} \\
e_{1}=\frac{\left\|\boldsymbol{c}_{13,1}\right\|}{\left|\mu_{13,1}\right|} \\
f_{\mathrm{B}}=\arctan \left(\frac{\hat{\boldsymbol{i}}_{c_{13,1}} \times \hat{\boldsymbol{i}}_{r_{\mathrm{B}}} \cdot \hat{\boldsymbol{i}}_{h_{13}}}{\hat{\boldsymbol{i}}_{c_{13,1}} \cdot \hat{\boldsymbol{i}}_{r_{\mathrm{B}}}}\right)
\end{gathered}
$$

Next to be investigated is the relationship between the maneuver time $\Delta t_{1}$ and the states of point $\mathrm{C}$. This process involves solving the anomaly angles and solving the Kepler's equation. Different types of the conic section use different formulas. Depending on the values of $\mu_{13,1}$ and $a_{13,1}$, the conic-section type of the phase 1 trajectory can be elliptical, parabolic, attractive hyperbolic, and repulsive hyperbolic. Here, we take the repulsive hyperbola as an example to show the calculation steps.

Giving $\Delta t_{1}$, the following procedure shows the steps to find $\boldsymbol{r}_{\mathrm{C}}$ and $\dot{\boldsymbol{r}}_{\mathrm{C}}$. The hyperbolic anomaly of point $\mathrm{B}$ is

$$
H_{\mathrm{B}}=2 \operatorname{arctanh}\left(\sqrt{\frac{e_{1}-1}{e_{1}+1}} \cdot \tan \frac{f_{\mathrm{B}}}{2}\right)
$$

The mean anomaly of point $\mathrm{B}$ is

$$
N_{B}=e_{1} \sinh H_{B}+H_{B}
$$

The mean anomaly of point $\mathrm{C}$ is

$$
N_{\mathrm{C}, 1}=N_{\mathrm{B}}+\sqrt{-\frac{\mu_{13,1}}{a_{13,1}^{3}}} \Delta t_{1}
$$

The hyperbolic anomaly of point $\mathrm{C}$ is obtained by numerically solving the transcendental equation

$$
N_{\mathrm{C}, 1}=e_{1} \sinh H_{\mathrm{C}, 1}+H_{\mathrm{C}, 1}
$$

The true anomaly of point $\mathrm{C}$ in phase 1 is

$$
f_{\mathrm{C}, 1}=2 \arctan \left(\sqrt{\frac{e_{1}+1}{e_{1}-1}} \tanh \frac{H_{\mathrm{C}, 1}}{2}\right)
$$

The radius of point $\mathrm{C}$ is

$$
r_{\mathrm{C}}=\frac{h_{13}^{2}}{\mu_{13,1}\left(e_{1} \cos f_{\mathrm{C}, 1}-1\right)}
$$

The Lagrange coefficients are

$$
F_{\mathrm{C}, 1}=1+\frac{a_{1}}{r_{\mathrm{B}}}\left(\cos \Delta H_{1}-1\right)
$$

$$
G_{\mathrm{C}, 1}=t_{1}+\sqrt{-\frac{a_{1}^{3}}{\mu_{13,1}}}\left(\sinh \Delta H_{1}-\Delta H_{1}\right)
$$

$$
\begin{gathered}
\dot{F}_{\mathrm{C}, 1}=\frac{\sqrt{-\mu_{13,1} a_{13,1}}}{r_{\mathrm{B}} r_{\mathrm{C}}} \sinh \Delta H_{1} \\
\dot{G}_{\mathrm{C}, 1}=1+\frac{a_{13,1}}{r_{\mathrm{C}}}\left(\cosh \Delta H_{1}-1\right)
\end{gathered}
$$

where $\Delta H_{1}=H_{\mathrm{C}, 1}-H_{\mathrm{B}}$. The states at point $\mathrm{C}$ are

$$
\boldsymbol{r}_{\mathrm{C}}=F_{\mathrm{C}, 1} \boldsymbol{r}_{\mathrm{B}}+G_{\mathrm{C}, 1} \dot{\boldsymbol{r}}_{\mathrm{B}}
$$

$$
\dot{\boldsymbol{r}}_{\mathrm{C}}=\dot{F}_{\mathrm{C}, 1} \boldsymbol{r}_{\mathrm{B}}+\dot{G}_{\mathrm{C}, 1} \dot{\boldsymbol{r}}_{\mathrm{B}}
$$

Note that the states of point $\mathrm{C}$ are obtained based on the provided value of $\Delta t_{1}$. Thus, $\boldsymbol{r}_{\mathrm{C}}$ and $\dot{\boldsymbol{r}}_{\mathrm{C}}$ are functions of $\Delta t_{1}$. Once the states of point $\mathrm{C}$ are known, the procedure developed in the last section can be used to obtain the phase 2 trajectory, which satisfies the requirement in Eq. (49). In the following, we are going to illustrate the way to find the phase 2 trajectory that satisfies Eq. (50).

The Lagrange coefficient of point $\mathrm{D}$ is

$$
F_{\mathrm{D}}=\frac{\boldsymbol{r}_{\mathrm{D}} \times \dot{\boldsymbol{r}}_{\mathrm{C}}}{\boldsymbol{r}_{\mathrm{C}} \times \dot{\boldsymbol{r}}_{\mathrm{C}}}
$$

The effective gravitational coefficient of phase 2 is

$$
\mu_{2}=\frac{\left(1-F_{\mathrm{D}}\right) h_{13}^{2}}{\left(1-\cos \Delta f_{2}\right) r_{\mathrm{D}}}
$$

where $\Delta f_{2}=\gamma-\Delta f_{1}=\gamma-\left(f_{\mathrm{C}, 1}-f_{\mathrm{B}}\right)$, and $r_{\mathrm{D}}=r_{13}^{*}$ is the desired radius of point $\mathrm{D}$. The semimajor axis of phase 2 is

$$
a_{2}=\frac{r_{\mathrm{C}} \mu_{2}}{2 \mu_{2}-r_{\mathrm{C}} v_{\mathrm{C}}^{2}}
$$

where $v_{\mathrm{C}}=\left\|\dot{\boldsymbol{r}}_{\mathrm{C}}\right\|$ is the magnitude of the velocity at point $\mathrm{C}$. The eccentricity vector is

$$
\boldsymbol{c}_{2}=\dot{\boldsymbol{r}}_{\mathrm{C}} \times \boldsymbol{h}_{13}-\mu_{2} \frac{\boldsymbol{r}_{\mathrm{C}}}{r_{\mathrm{C}}}
$$

The true anomaly of point $\mathrm{C}$ in phase 2 is

$$
f_{\mathrm{C}, 2}=\arctan \left(\frac{\hat{\boldsymbol{i}}_{c_{2}} \times \hat{\boldsymbol{i}}_{r_{\mathrm{C}}} \cdot \hat{\boldsymbol{i}}_{h}}{\hat{\boldsymbol{i}}_{c_{2}} \cdot \hat{\boldsymbol{i}}_{r_{\mathrm{C}}}}\right)
$$

Now, we are going to use Kepler's equation to calculate $\boldsymbol{r}_{\mathrm{D}}$ corresponding to $\Delta t_{2}=t^{*}-\Delta t_{1}$. Different types of orbits have different formulas toward the Kepler's equation. In the following, the hyperbolic orbit type is taken as an example. The hyperbolic anomaly of point $\mathrm{C}$ is 
Table 3 Calculations of $F$ and $G$ coefficients in different situations

\begin{tabular}{lccl}
\hline \hline$\mu$ & $a$ & Trajectory type & \multicolumn{1}{c}{$F$ and $G$ coefficients } \\
\hline$\mu>0$ & $a>0$ & Ellipse & $F=1-\frac{\mu r_{1}}{h^{2}}(1-\cos \Delta f)$ \\
& & & \\
& & & \\
& $a<0$ & Attractive hyperbola & $r_{0} r_{1} \Delta f$ \\
$h$ & $\dot{F}=\frac{\mu(1-\cos \Delta f)}{h \sin \Delta f}\left[\frac{\mu(1-\cos \Delta f)}{h^{2}}-\frac{1}{r_{0}}-\frac{1}{r_{1}}\right]$ \\
& & & $\dot{G}=1-\frac{\mu r_{0}}{h^{2}}(1-\cos \Delta f)$ \\
$\mu<0$ & $a>0$ & Repulsive hyperbola & $F=1+\frac{a}{r}(\cos \Delta H-1)$ \\
& & & $G=t+\sqrt{-\frac{a^{3}}{\mu}}(\sinh \Delta H-\Delta H)$ \\
& & & $\dot{F}=\frac{\sqrt{-\mu a}}{r_{0} r_{1}} \sinh \Delta H$ \\
& & $\dot{G}=1+\frac{a}{r_{1}}(\cosh \Delta H-1)$ \\
\hline \hline
\end{tabular}

$$
H_{\mathrm{C}, 2}=2 \operatorname{arctanh}\left(\sqrt{\frac{e_{2}-1}{e_{2}+1}} \cdot \tan \frac{f_{\mathrm{C}, 2}}{2}\right)
$$

where $e_{2}=\left\|c_{2}\right\| /\left|\mu_{2}\right|$. The mean anomaly at point $\mathrm{C}$ is

$$
N_{\mathrm{C}, 2}=e \sinh H_{\mathrm{C}, 2}+H_{\mathrm{C}, 2}
$$

The desired mean anomaly at point $\mathrm{D}$ is

$$
N_{\mathrm{D}}=N_{\mathrm{C}, 2}+n \Delta t_{2}
$$

where $n_{2}=\sqrt{-\mu_{2} / a_{2}^{3}}$, and $\Delta t_{2}=t^{*}-\Delta t_{1}$. The hyperbolic anomaly of point $\mathrm{D}$ is obtained by numerically solving the transcendental equation

$$
N_{\mathrm{D}}=e_{2} \sinh H_{\mathrm{D}}+H_{\mathrm{D}}
$$

The change of hyperbolic anomaly of phase 2 is

$$
\Delta H_{2}=H_{\mathrm{D}}-H_{\mathrm{C}, 2}
$$

The $F$ and $G$ coefficients are determined by $\Delta H_{2}$ :

$$
\begin{gathered}
F_{\mathrm{D}}=1+\frac{a_{2}}{r_{\mathrm{C}}}\left(\cos \Delta H_{2}-1\right) \\
G_{\mathrm{D}}=t_{1}+\sqrt{-\frac{a_{2}^{3}}{\mu_{2}}}\left(\sinh \Delta H_{2}-\Delta H_{2}\right)
\end{gathered}
$$

Then, the position vector at point $\mathrm{D}$ is obtained through

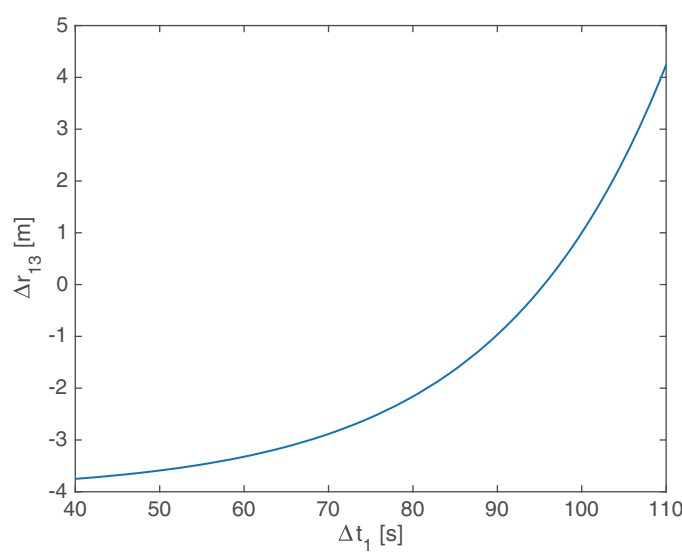

a) $\Delta t_{\mathrm{I}}-\Delta r$ while the types of the trajectory remains consistent

$$
\boldsymbol{r}_{\mathrm{D}}=F_{\mathrm{D}} \boldsymbol{r}_{\mathrm{C}}+G_{\mathrm{D}} \dot{\boldsymbol{r}}_{\mathrm{C}}
$$

Table 3 gives the calculations of the $F$ and $G$ coefficients for different types of orbit.

Assuming that $Q_{13,1}$ and $\Delta t_{1}$ are given, the procedure in Eqs. (5680 ) finds the trajectory that satisfies the maneuver time requirement in Eq. (50). The next problem is to find the solution that satisfies both the maneuver time requirement in Eq. (50) and the final location requirement in Eq. (49).

The procedure in Eqs. (56-80) reveals that the final position $\boldsymbol{r}_{\mathrm{D}}$ is determined by $Q_{13,1}$ and $\overline{\Delta t_{1}}$ :

$$
\boldsymbol{r}_{\mathrm{D}}=f\left(\Delta t_{1}, Q_{13,1}\right)
$$

This function is not a fundamental function because there is a transcendental equation when calculating the final vector $\boldsymbol{r}_{\mathrm{D}}$. As this paper assumes that $Q_{13,1}$ is given, then numerically search the solution $\Delta t_{1}^{*}$ that satisfies Eq. (49). Defining a scalar error function as

$$
\Delta r=r_{\mathrm{D}} \cdot r_{\mathrm{D}}^{*}-r_{\mathrm{D}}^{* 2}
$$

The objective of the numerical algorithm is to find the value of $\Delta t_{1}$ such that $\Delta r \rightarrow 0$. The combination of the types of two-phase trajectory can lead to four cases: 1) both ellipse; 2) ellipse and hyperbola; 3) hyperbola and ellipse; or 4) both hyperbola. During the numerical searching process, if the combination of the trajectory type remains in case 1 or 4 , the curve of $\Delta t_{1}-\Delta r$ is continuous and smooth, as shown in Fig. 7a. The secant method is applicable to solve the solution $\Delta t_{1}^{*}$ in this case. The corresponding procedure of the numerical algorithm is given by

$$
\Delta t_{1}(k)=\Delta t_{1}(k-1)-\alpha \frac{\Delta t_{1}(k-1)-\Delta t_{1}(k-2)}{\Delta r(k-1)-\Delta r(k-2)} \Delta r(k-1)
$$

where $0<\alpha<1$ is the coefficient used to reduce the sensitivity of the algorithm.

There exists a possibility that the combination of the trajectory type changes when sweeping $\Delta t_{1}$, as shown in Fig. $7 \mathrm{~b}$. On the left-hand side of the switch point, the combination is "both ellipse," and on the other side, the combination is "ellipse and hyperbola." Moreover, the solution $\Delta t_{1}^{*}$ is very close to the switch point. In this case, the secant method is very sensitive around the solution. It would cause severe oscillations of the numerical searching process, as illustrated in

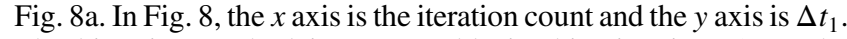
The bisection method is more stable in this situation. Once the oscillation phenomenon is detected, the secant method is terminated and the bisection method is engaged. The range of the solution is given by the largest range of $\Delta t_{1}$ during the oscillation, as shown in Fig. 8b.

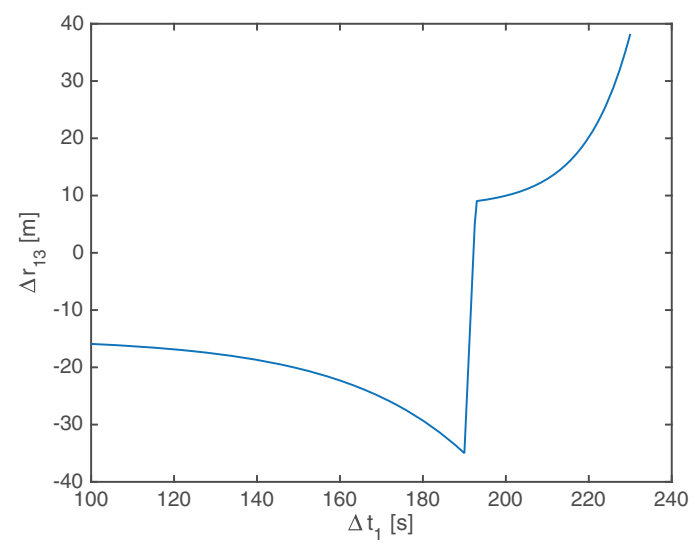

b) $\Delta t_{\mathrm{I}}-\Delta r$ while the types of the trajectory changes

Fig. $7 \Delta t_{1}-\Delta r$ curve in different situations. 


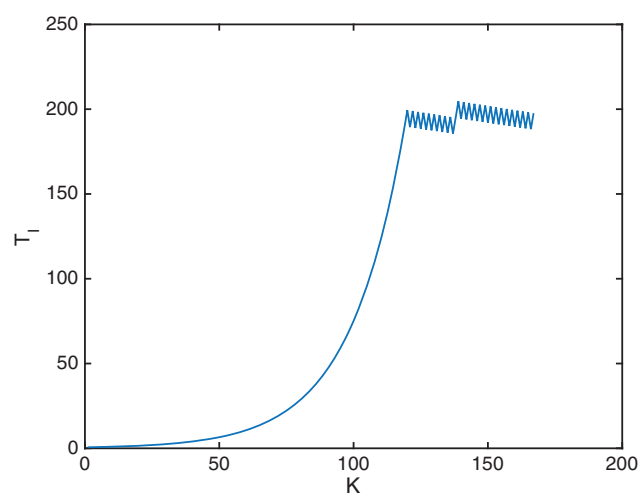

a) Oscillating error function

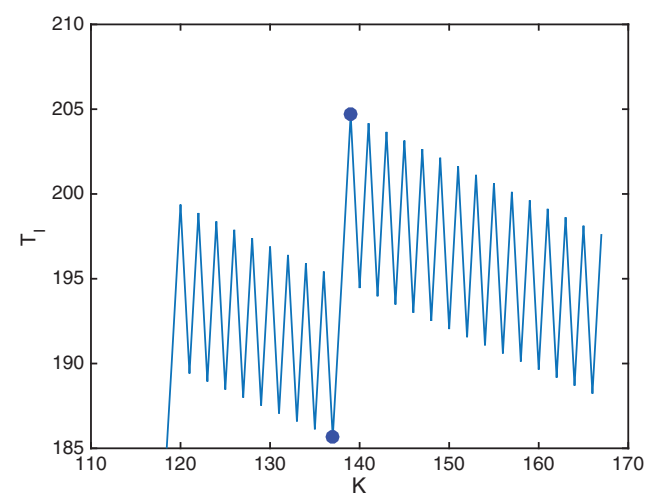

b) The searching range of the bisection method

Fig. 8 Oscillation phenomenon using the SECANT method to solve $\Delta t_{1}^{*}$.

Once $\Delta t_{1}^{*}$ is obtained, the flight time of the second phase is given by

$$
\Delta t_{2}^{*}=t^{*}-\Delta t_{1}^{*}
$$

Using the procedure determining the single-phase trajectory, the charge product $Q_{13,2}$ is uniquely determined.

So far, all of the parameters determining the entire maneuver trajectory $\left[t^{*}, \Delta t, \Delta t_{1}^{*}, Q_{13,1}, \Delta t_{2}^{*}, Q_{13,2}\right]$ are obtained. The procedure of the reconfiguration strategy to achieve the control objective is summarized as follows:

1) Choose two spacecraft to be the subsystem such that there exists at least one positive solution to Eq. (20). (If there is no such subsystem, the initial conditions of the system need to be tuned. The adjustment of the initial condition will be discussed in the next section.)

2) Solve Eq. (20) analytically and find a positive real solution $t^{*}$.

3) Obtain the maneuver time $\Delta t$ of the one-phase trajectory using the procedure listed in Table 2.

4) Check whether $\Delta t=t^{*}$ is satisfied. If it is satisfied, stop. The control objective can be realized by using the one-phase trajectory strategy. If not, go to the next step.

5) Choose a value of $Q_{13,1}$, and then numerically solve $\Delta t_{1}^{*}$ such that both Eqs. (49) and (50) are satisfied.

Note that there is one degree of freedom in the two-phase maneuver trajectory design. This flexibility can be used to find the optimal solution by a certain well-defined cost function. The optimization problem is beyond the scope of this paper.

\section{Adjustment of the Improper Initial Conditions}

The last two sections develop the maneuver trajectories based on the fact that, by choosing different combinations of the subsystem, there exists a combination such that Eq. (20) has at least one positive solution. In the case that there are no combinations of the subsystem

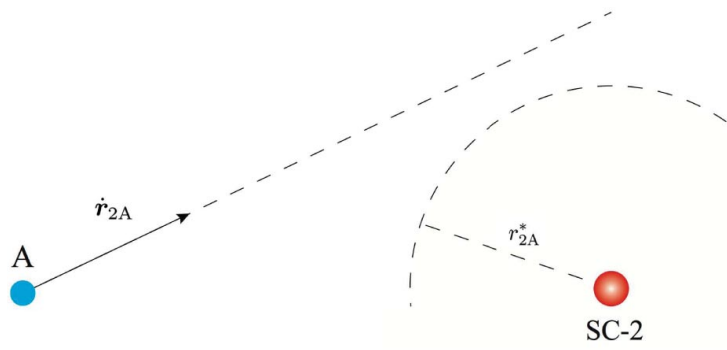

a) Equation (20) has imaginary solutions with positive real part that make Eq. (20) have positive solutions, the two-phase maneuver trajectories developed previously cannot be applied to achieve the reconfiguration. The cases of the solutions to Eq. (20) are summarized in Table 1 and Fig. 4 .

In the case that there are no positive solutions to Eq. (20), there is a special situation that the solutions to Eq. (20) are a pair of imaginary numbers with a positive real part. The corresponding scenario is illustrated in Fig. 9a. In this case, it is possible to adjust the initial conditions such that Eq. (20) has at least one positive solution. This adjustment strategy is investigated in this section.

In Fig. $9 \mathrm{~b}, \dot{\boldsymbol{r}}_{2 \mathrm{~A}}$ is decomposed along the radial direction and the tangential direction of $\boldsymbol{r}_{2 \mathrm{~A}}$. It can be seen that the reason why the trajectory of point A deviates the circle centered at SC-2 is that the tangential component of $\dot{\boldsymbol{r}}_{2 A}$ is too large. Now, let us investigate the tangential component of point A, SC-1, and SC-3. Projecting $\dot{\boldsymbol{r}}_{12}$ and $\dot{\boldsymbol{r}}_{23}$ to the direction defined by $\boldsymbol{\tau}_{v A}$ yields

$$
\begin{gathered}
\boldsymbol{\tau}_{v_{A}}=\dot{\boldsymbol{R}}_{\mathrm{A}}-\frac{\dot{\boldsymbol{R}}_{\mathrm{A}} \cdot \boldsymbol{r}_{\mathrm{A} 2}}{\left\|\boldsymbol{r}_{\mathrm{A} 2}\right\|} \cdot \frac{\boldsymbol{r}_{\mathrm{A} 2}}{\left\|\boldsymbol{r}_{\mathrm{A} 2}\right\|} \\
\boldsymbol{\tau}_{v_{1}}=\dot{\boldsymbol{R}}_{1}-\frac{\dot{\boldsymbol{R}}_{1} \cdot \boldsymbol{r}_{\mathrm{A} 2}}{\left\|\boldsymbol{r}_{\mathrm{A} 2}\right\|} \cdot \frac{\boldsymbol{r}_{\mathrm{A} 2}}{\left\|\boldsymbol{r}_{\mathrm{A} 2}\right\|} \\
\boldsymbol{\tau}_{v_{3}}=\dot{\boldsymbol{R}}_{3}-\frac{\dot{\boldsymbol{R}}_{3} \cdot \boldsymbol{r}_{\mathrm{A} 2}}{\left\|\boldsymbol{r}_{\mathrm{A} 2}\right\|} \cdot \frac{\boldsymbol{r}_{\mathrm{A} 2}}{\left\|\boldsymbol{r}_{\mathrm{A} 2}\right\|}
\end{gathered}
$$

Substituting Eq. (ㅁ) into Eq. (모) yields

$$
\boldsymbol{\tau}_{v_{A}}=\frac{m_{1} \boldsymbol{\tau}_{v_{1}}+m_{3} \boldsymbol{\tau}_{v_{3}}}{m_{1}+m_{3}}
$$

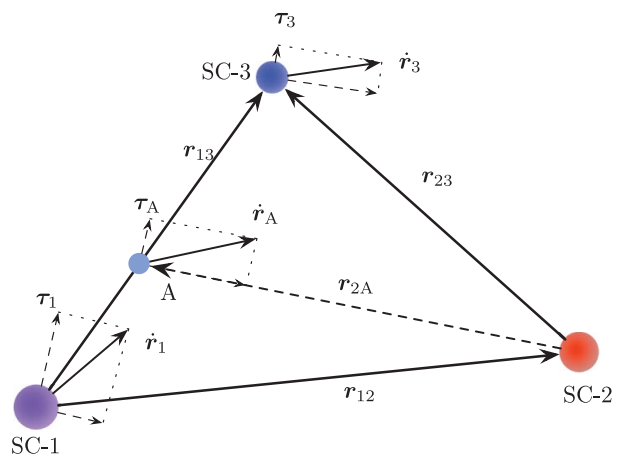

b) Decomposition of the velocities 
The magnitude of $\boldsymbol{\tau}_{v_{A}}$ satisfies

$$
\tau_{v_{A}}=\frac{m_{1} \tau_{v_{1}}+m_{3} \tau_{v_{3}}}{m_{1}+m_{3}}
$$

As mentioned previously, due to the fact that the magnitude $\tau_{v_{A}}$ is too large, the trajectory of point A deviates from the effective circle. One way to correct this is to introduce another phase (an adjustment phase) to reduce the variable $\tau_{v_{A}}$. In the adjustment phase, we still propose the conic-section trajectory approach to finish the job. SC-2 and another spacecraft are grouped as a subsystem, and they are both charged. The other spacecraft in the subsystem is determined by comparing the contributions to $\tau_{v_{A}}$ as follows:

1) If $\tau_{v_{1}}>\tau_{v_{A}}$, choose SC-1 and SC- 2 as the preadjusted subsystem.

2) If $\tau_{v_{3}}>\tau_{v_{A}}$, choose SC-3 and SC-2 as the preadjusted subsystem.

Without loss of generality, SC-1 and SC-2 are taken as the preadjusted subsystem to show the calculation procedure. The adjustment phase is illustrated in Fig. 10. At time $t_{\text {ad }}$, the velocity of SC-2 is

$$
\dot{\boldsymbol{R}}_{2}\left(t_{\mathrm{ad}}\right)=\dot{\boldsymbol{R}}_{2}(0)+\Delta \dot{\boldsymbol{R}}_{2}
$$

In the adjustment phase, there are no external forces acting on the SC-1 and SC-2 preadjusted subsystem; then,

$$
\frac{m_{1} \dot{\boldsymbol{R}}_{1}\left(t_{\mathrm{ad}}\right)+m_{2} \dot{\boldsymbol{R}}_{2}\left(t_{\mathrm{ad}}\right)}{m_{1}+m_{2}}=\frac{m_{1} \dot{\boldsymbol{R}}_{1}(0)+m_{2} \dot{\boldsymbol{R}}_{2}(0)}{m_{1}+m_{2}}
$$

Substituting Eq. (0) into Eq. (1) yields

$$
\dot{\boldsymbol{R}}_{1}\left(t_{\mathrm{ad}}\right)=\frac{m_{1} \dot{\boldsymbol{R}}_{1}(0)-m_{2} \Delta \dot{\boldsymbol{R}}_{2}}{m_{1}}
$$

Then, the relative position vectors $\dot{\boldsymbol{r}}_{12}\left(t_{\mathrm{ad}}\right)$ and $\dot{\boldsymbol{r}}_{\mathrm{A} 2}\left(t_{\mathrm{ad}}\right)$ are

$$
\begin{gathered}
\dot{\boldsymbol{r}}_{12}\left(t_{\mathrm{ad}}\right)=\dot{\boldsymbol{R}}_{2}\left(t_{\mathrm{ad}}\right)-\dot{\boldsymbol{R}}_{1}\left(t_{\mathrm{ad}}\right)=\dot{\boldsymbol{r}}_{12}(0)+\frac{m_{1}+m_{2}}{m_{1}} \Delta \dot{\boldsymbol{R}}_{2} \\
\dot{\boldsymbol{r}}_{\mathrm{A} 2}\left(t_{\mathrm{ad}}\right)=\dot{\boldsymbol{R}}_{2}\left(t_{\mathrm{ad}}\right)-\dot{\boldsymbol{R}}_{\mathrm{A}}\left(t_{\mathrm{ad}}\right)=\dot{\boldsymbol{r}}_{\mathrm{A} 2}(0)+\frac{m_{1}+m_{2}+m_{3}}{m_{1}+m_{3}} \Delta \dot{\boldsymbol{R}}_{2}
\end{gathered}
$$

Note that $\dot{\boldsymbol{r}}_{2 \mathrm{~A}}\left(t_{\mathrm{ad}}\right)=\dot{\boldsymbol{r}}_{2 \mathrm{~A}}(0)+\Delta \dot{\boldsymbol{r}}_{2 \mathrm{~A}}$; thus, the relationship between $\Delta \dot{\boldsymbol{r}}_{2 \mathrm{~A}}$ and $\Delta \dot{\boldsymbol{r}}_{12}$ is

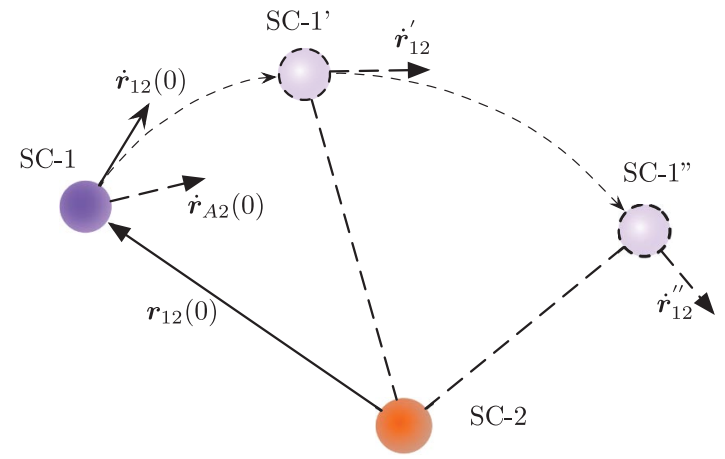

Fig. 10 Elliptic preadjusting trajectory.

$$
\Delta \dot{\boldsymbol{r}}_{2 \mathrm{~A}}=-\frac{m_{1}\left(m_{1}+m_{2}+m_{3}\right)}{\left(m_{1}+m_{2}\right)\left(m_{1}+m_{3}\right)} \Delta \dot{\boldsymbol{r}}_{12}
$$

As shown in Fig. 11 , by properly designing $\Delta \dot{\boldsymbol{r}}_{12}$, the trajectory of point $\mathrm{A}$ can be adjusted such that point $\mathrm{A}$ will cross the effective sphere centered at SC-2, which can be formulated as

$$
d_{\mathrm{os}} \leq r_{2 \mathrm{~A}}^{*}
$$

The adjustment process is summarized as follows:

1) Set the charge product $Q_{\mathrm{ad}}$ and the adjustment time $t_{\mathrm{ad}}$ of the preadjusting subsystem in the total preadjusting process.

$Q_{\text {ad }}$ should satisfy the collision avoidance and charge saturation requirement. To minimize the time duration of the preadjusting process, it is recommended that $\left|Q_{\mathrm{ad}}\right|=Q_{\max }$. If $\tau_{v_{1}}>\tau_{v_{A}}$, set the charge product as $Q_{\mathrm{ad}}=Q_{12}=q_{1} q_{2}$. If $\tau_{v_{3}}>\tau_{v_{4}}$, set the charge product as $Q_{\mathrm{ad}}=Q_{23}=q_{2} q_{3}$. The range of $t_{\mathrm{ad}}$ is $\left(0, t^{*}\right)$.

2) Solve the parameters of the preadjusting phase, and obtain $\dot{\boldsymbol{r}}_{2 \mathrm{~A}}\left(t_{\mathrm{ad}}\right)$.

3) Calculate the offset distance $d_{\mathrm{os}}$ from SC2 to $\dot{\boldsymbol{r}}_{2 \mathrm{~A}}$. If $d_{\mathrm{os}} \leq r_{2 \mathrm{~A}}^{*}$, end. Otherwise, increase the value of $t_{\mathrm{ad}}$ and go to step 2. If $t_{\mathrm{ad}} \geq t^{*}$ and $d_{\mathrm{os}}>r_{2 \mathrm{~A}}^{*}$, the reconfiguration mission cannot be accomplished using the trajectory programming method in this paper.

Figure 12 illustrates the relationship between $Q_{\mathrm{ad}}, t_{\mathrm{ad}}$, and $d_{\mathrm{os}}$. It can be seen that the range of $d_{\mathrm{os}}$ is $[0,50+] \mathrm{m}$. As shown in Fig. 12b, which indicates the regions of the solutions to Eq. (96) with a green color, giving a value of $Q_{\mathrm{ad}}$, there are multiple regions of $t_{\mathrm{ad}}$ that satisfy Eq. (96). From the bottom to the top, the first region corresponds to an adjusting trajectory that is a section of an ellipse. The second region represents solutions with the adjusting trajectory that is composed of a complete ellipse and a section of the ellipse. The first region is the most important. As the magnitude of $Q_{\mathrm{ad}}$ increases, the region of the solutions decreases. The bottom line of the region corresponds to the solutions that satisfy

$$
d_{\mathrm{os}}=r_{2 \mathrm{~A}}^{*}
$$

\section{Numerical Simulations}

Numerical simulations are presented in this section to illustrate the performance of the reconfiguration strategy. The desired configuration is a triangle defined by the three side lengths $\left[l_{12}^{*}, l_{23}^{*}, l_{13}^{*}\right]$. The masses of the three spacecraft are

$$
m_{1}=m_{2}=m_{3}=50 \mathrm{~kg}
$$

In the following simulation cases, the initial conditions and the expected configurations are set to be exactly the same as the simulation examples in [15].

\section{A. Large Effort Maneuver}

The initial positions and velocities of the three spacecraft are

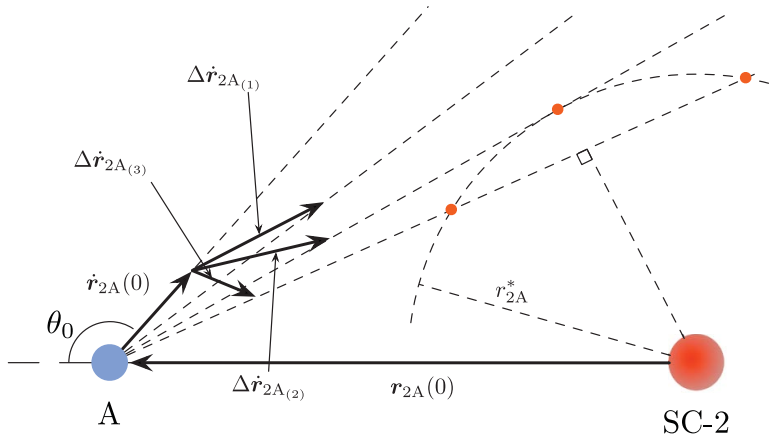

Fig. 11 Effects of different $\Delta \dot{r}_{2 A}$. 


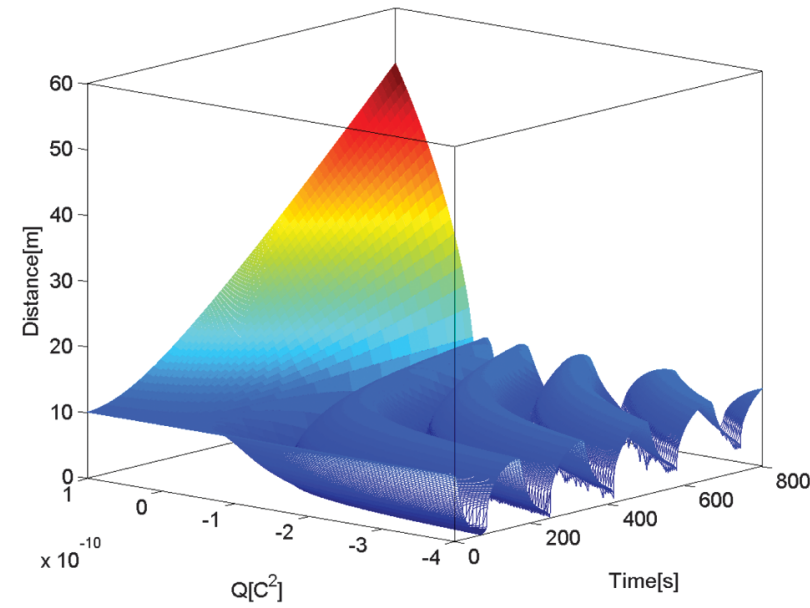

a) Three-dimensional figure

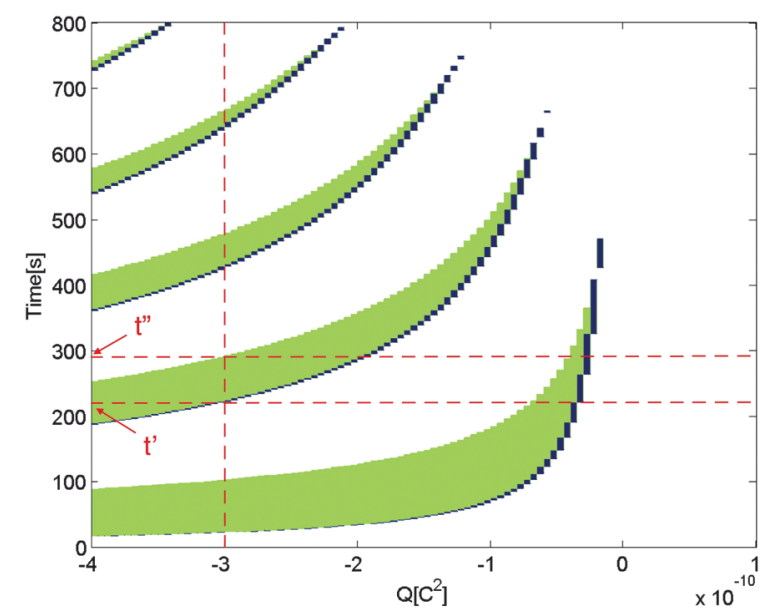

b) Contour plot

Fig. 12 Relationship between $Q_{\text {ad }}, t_{\text {ad }}$, and $d_{\text {os }}$.

$$
\left\{\begin{array} { l } 
{ \boldsymbol { R } _ { 1 } = [ 9 , - 2 , 0 ] \mathrm { m } ; } \\
{ \boldsymbol { R } _ { 2 } = [ 0 , - 4 , 0 ] \mathrm { m } ; } \\
{ \boldsymbol { R } _ { 3 } = [ - 2 , - 2 , 0 ] \mathrm { m } ; }
\end{array} \quad \left\{\begin{array}{l}
\dot{\boldsymbol{R}}_{1}=[0,0.01,0] \mathrm{m} / \mathrm{s} ; \\
\dot{\boldsymbol{R}}_{2}=[0,0,0] \mathrm{m} / \mathrm{s} ; \\
\dot{\boldsymbol{R}}_{3}=[0,-0.01,0] \mathrm{m} / \mathrm{s} ;
\end{array}\right.\right.
$$

The expected triangle of the virtual structure is defined by the separation distances

$$
\boldsymbol{l}=\left[l_{12}^{*}, l_{13}^{*}, l_{23}^{*}\right]=[6,5,7] \mathrm{m}
$$

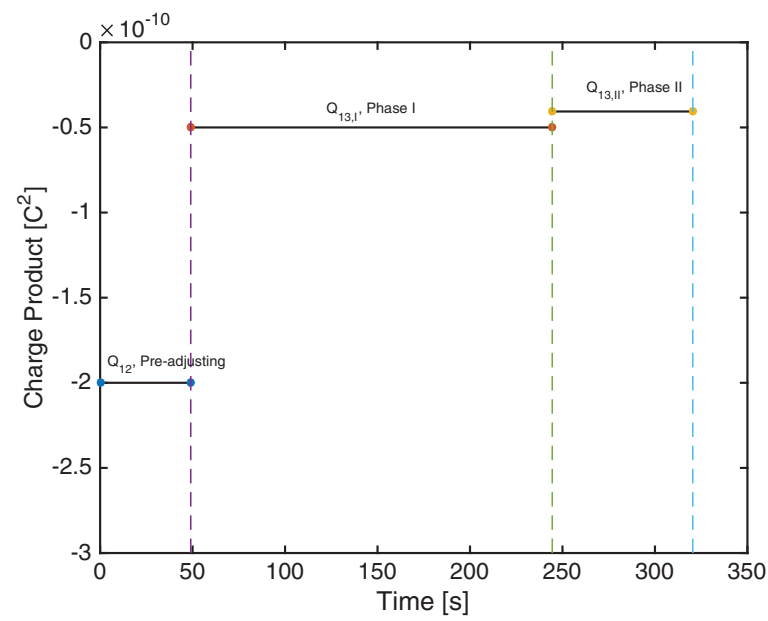

a) Charge histories

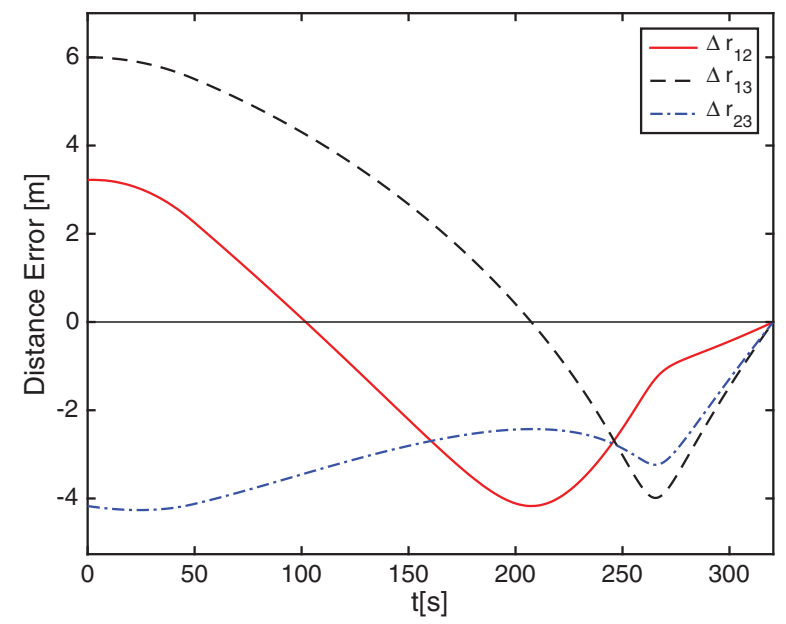

c) The distance errors
In this example, the initial errors of the formation are relatively large, which would result in the large control efforts. One may find that Eq. (20) does not have positive solutions based on the given initial conditions. So, the adjustment of the initial conditions is implemented. SC-1 and SC-2 are chosen as the preadjusting subsystem. The two free variables of the adjustment are set to be

$$
Q_{\mathrm{ad}}=-2 \times 10^{-10} \mathrm{C}^{2}, \quad t_{\mathrm{ad}}=49 \mathrm{~s}
$$

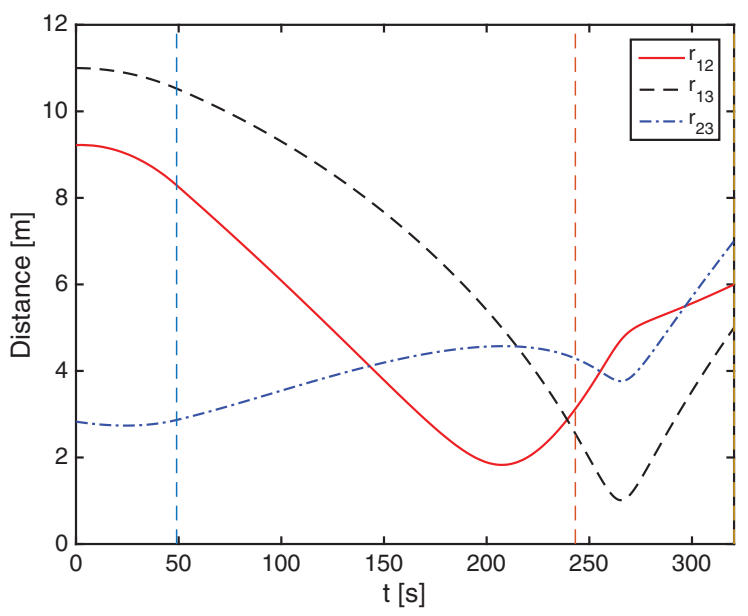

b) The separation distances

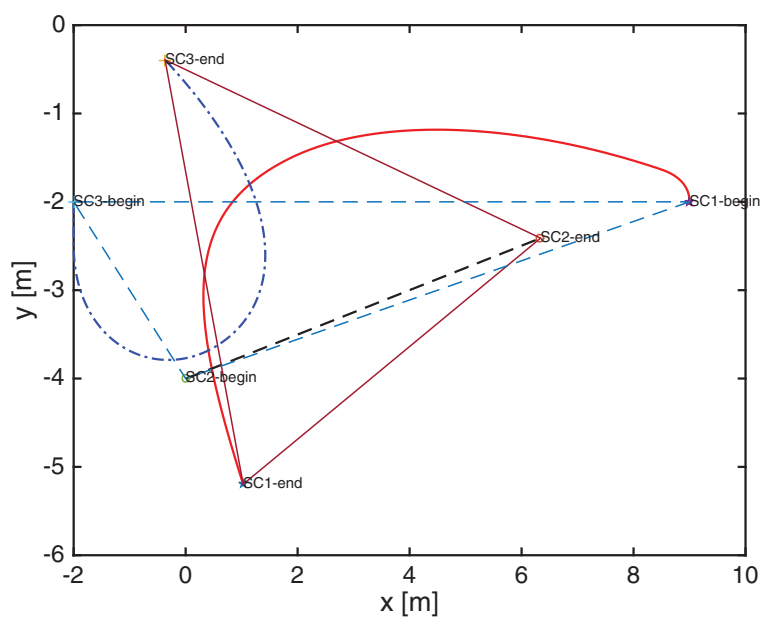

d) The trajectories of the three spacecraft in the inertial frame

Fig. 13 Simulation results of the large effort maneuver. 
The solutions to Eq. (20) are

$$
t_{1}^{*}=-85.0 \mathrm{~s}, \quad t_{2}^{*}=271.5 \mathrm{~s}
$$

The unique positive solution is $t^{*}=271.5 \mathrm{~s}$. The maneuver time of the single-phase trajectory is $\Delta t=349.4 \mathrm{~s}$. Because $\Delta t>t^{*}$, after the adjustment phase, the two-phase trajectory is chosen to achieve the reconfiguration.

Setting the charge product of phase 1 as $Q_{13,1}=-5 \times 10^{-11} \mathrm{C}^{2}$, the remaining variables are

$\Delta t_{1}^{*}=194.2 \mathrm{~s}, \quad Q_{13,2}=-4.05 \times 10^{-11} \mathrm{C}^{2}, \quad \Delta t_{2}^{*}=77.3 \mathrm{~s}$

The simulation results are illustrated in Fig. 13. Figure 13a is the history of the nonzero charge product. During the adjustment phase, $Q_{12}=-2 \times 10^{-10} \mathrm{C}^{2}$ and $q_{3}=0$. The charge product of the adjustment phase is set to be large such that the system can reach the proper state in a short period of time. The entire history of the nonzero charge product is composed of three horizontal lines. This type of control input is easy to implement in real situations.

Figure $13 \mathrm{~b}$ shows the distance histories, and Fig. 13c shows the error histories of the separation distances. At the moment when $t=t_{\text {ad }}+t^{*} \approx 320.5 \mathrm{~s}$, the distances of the triangle are

$$
\left[l_{12}, l_{13}, l_{23}\right]=[5.9984,5.0020,7.0045] \mathrm{m}
$$

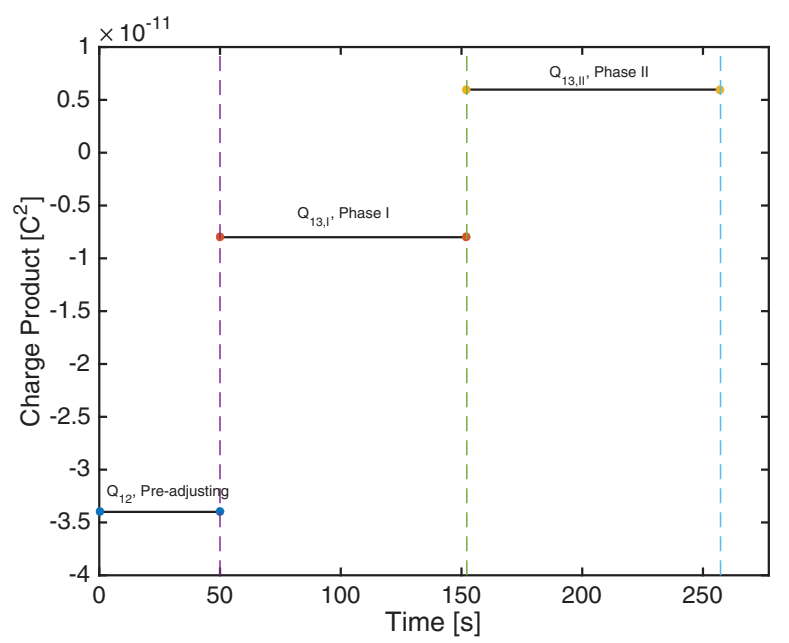

a) Charge histories

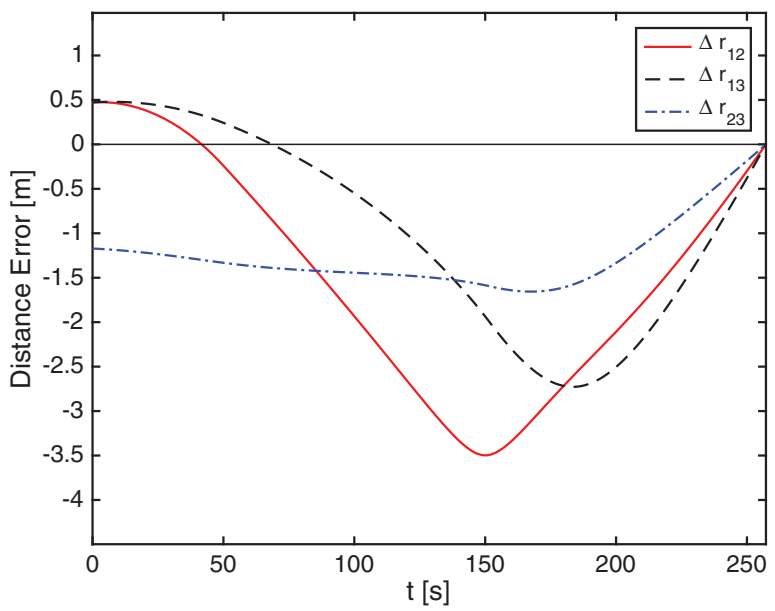

c)The distance errors
The small errors are due to the limited precision of the numerical routine solving for $\Delta t_{1}^{*}$. Figure $13 \mathrm{~d}$ shows the trajectories of the three spacecraft. It can be seen that the trajectories of SC-1 and SC-3 are pretty curvy, but the trajectory of SC-2 is almost in a straight line. This is because SC- 2 is charged only in the adjustment phase and, during that phase, SC-1 lies almost on the initial velocity direction of SC-2.

\section{B. Small Effort Maneuver}

In this example, the initial positions and velocities of the three spacecraft are

$$
\left\{\begin{array} { l } 
{ \boldsymbol { R } _ { 1 } = [ 2 , 0 , 0 ] \mathrm { m } ; } \\
{ \boldsymbol { R } _ { 2 } = [ 0 , - 4 , 0 ] \mathrm { m } ; } \\
{ \boldsymbol { R } _ { 3 } = [ - 2 , - 2 , 0 ] \mathrm { m } ; }
\end{array} \quad \left\{\begin{array}{l}
\dot{\boldsymbol{R}}_{1}=[0,0.002,0] \mathrm{m} / \mathrm{s} ; \\
\dot{\boldsymbol{R}}_{2}=[0,0,0] \mathrm{m} / \mathrm{s} ; \\
\dot{\boldsymbol{R}}_{3}=[0,-0.002,0] \mathrm{m} / \mathrm{s} ;
\end{array}\right.\right.
$$

The desired configuration is given by the separation distances

$$
\boldsymbol{l}=\left[l_{12}^{*}, l_{13}^{*}, l_{23}^{*}\right]=[4,4,4] \mathrm{m}
$$

Note that the initial errors are smaller than those in the last simulation case. Similar to the last simulation case, in this example, Eq. (20) does not have positive solutions either. So, the adjustment of the initial conditions is implemented. SC- 1 and SC- 2 are chosen as the preadjusting subsystem. The two free variables of the adjustment phase are set to be

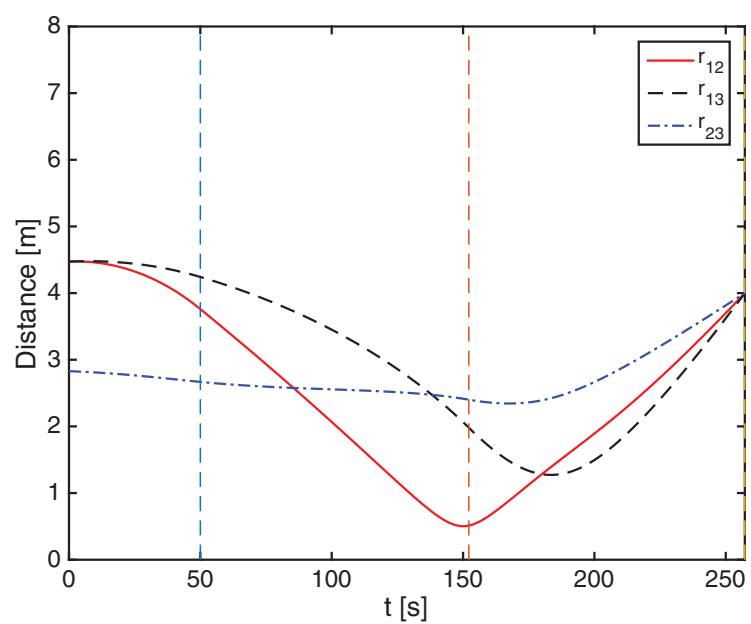

b)The separation distances

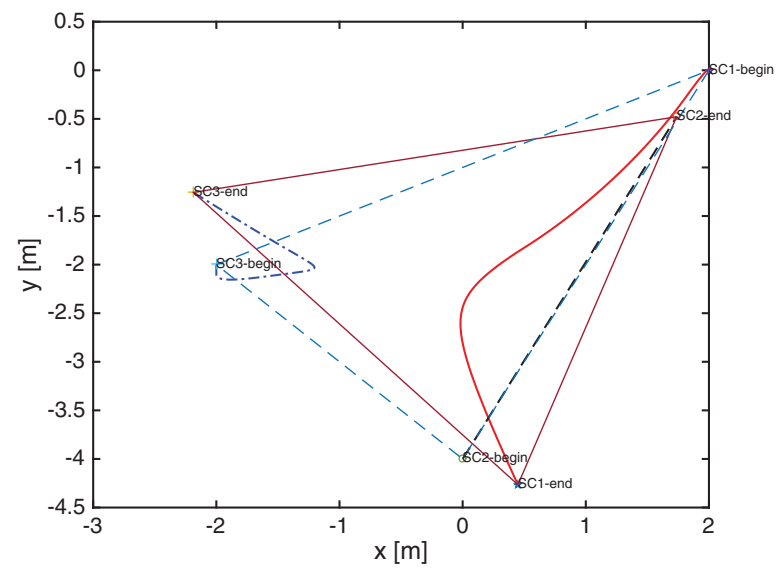

d) The trajectories of the three spacecraft in the inertial frame

Fig. 14 Simulation results of the small effort maneuver. 


$$
Q_{\mathrm{ad}}=-3.4 \times 10^{-11} \mathrm{C}^{2}, \quad t_{\mathrm{ad}}=50 \mathrm{~s}
$$

After the adjustment, there is only one positive solution to Eq. (20), which is $t^{*}=207.3 \mathrm{~s}$. The maneuver time of the single-phase trajectory is $\Delta t=430.1 \mathrm{~s}$. Because $\Delta t>t^{*}$, after the adjustment, the two-phase trajectory is required to achieve the control objective.

Setting the charge product of phase 1 as $Q_{13,1}=-8 \times 10^{-12} \mathrm{C}^{2}$, the corresponding parameters of the trajectory are

$$
\Delta t_{1}^{*}=102.2 \mathrm{~s}, \quad Q_{13,2}=6.0 \times 10^{-12} \mathrm{C}^{2}, \quad \Delta t_{2}^{*}=105.1 \mathrm{~s}
$$

Figure 14 illustrates the simulation results of this case. It can be seen from Fig. 14a that the nonzero charge product is negative during the adjustment phase and phase 1 , and it is positive during phase 2 . Figure $14 \mathrm{c}$ shows that, at time $t=t_{\mathrm{ad}}+t^{*} \approx 257.3 \mathrm{~s}$, the distance errors are almost zero. The final distances between the two spacecraft are

$$
\left[l_{12}, l_{13}, l_{23}\right]=[3.9997,3.9992,3.9999] \mathrm{m}
$$

In these two simulation cases, the maneuver trajectories are composed of three phases. During each phase, the charges of individual spacecraft remain constant. This strategy ensures that the control inputs are much smoother and much easier to be implemented in reality. Note that there are three degrees of freedom in the threephase reconfiguration maneuver trajectory design. These flexibilities can be used to find certain optimal solutions, which would be in future work beyond this paper.

\section{Conclusions}

This paper develops a trajectory programming strategy for the reconfiguration of a three-craft Coulomb formation. The entire trajectories are composed of multiple phases. During each phase, the charges of the individual spacecraft remain constant. In the general situation, the complete strategy is composed of three phases: a preadjusting phase, and two transition phases. The single-phase strategy is applicable only at certain occasional situations with strict requirements. The two-phase strategy is applicable if there exist positive solutions to the maneuver time requirement shown in Eq. (20). If the solutions to Eq. (20) are imaginary values with a positive real part, the initial conditions need to be adjusted. The preadjusting phase tunes the states of the system such that Eq. (20) has at least one positive solution. The resulting reconfiguration strategy has elegant control inputs. There are three extra degrees of freedom in determining the three-phase maneuver strategy, or one degree of freedom for the two-phase maneuver strategy if the solution exists. These flexibilities can be used to optimize the trajectories. The optimization of the trajectories will be in future work from this paper. It should also be noted that, in developing the strategy, the Debye shielding effect is not taken into consideration. To implement the maneuver strategy in the real space environment, a feedback controller should be engaged to compensate for the modeling errors brought by various sources such as the Debye shielding effect. Designing the feedback control with a reference trajectory input for the reconfiguration will be another direction for future work from this paper.

\section{Acknowledgment}

This research is supported by the National Natural Science Foundation (NSFC) of China: NSFC 61203198.

\section{References}

[1] King, L. B., Parker, G. G., Deshmukh, S., and Chong, J.-H., "Spacecraft Formation-Flying Using Inter-Vehicle Coulomb Forces," NASA/NASA Inst. for Advanced Concepts, Jan. 2002, http://www.niac.usra.edu/files/ studies/final_report/601King.pdf [retrieved 16 November 2015].

[2] Nishikawa, K., Plasma Physics, 3rd ed., Springer Series on Atomic, Optical, and Plasma Physics, Springer, New York, 2000, pp. 56-60.

[3] Romanelli, C. C., Natarajan, A., Schaub, H., Parker, G. G., and King, L. B., "Coulomb Spacecraft Voltage Study Due to Differential Orbital Perturbations," AAS Space Flight Mechanics Meeting, American Astronomical Soc. Paper 2006-123, Washington, D.C., Jan. 2006.

[4] Ren, W., and Beard, R. W., "Virtual Structure Based Spacecraft Formation Control with Formation Feedback," AIAA Guidance, Navigation, and Control Conference and Exhibit, AIAA Paper 20024693, Aug. 2002.

[5] Schaub, H., and Hussein, I., "Stability and Reconfiguration Analysis of a Circularly Spinning 2-Craft Coulomb Tether," IEEE Transactions on Aerospace and Electronic Systems, Vol. 38, No. 12, Oct. 2010, pp. $1675-1686$. doi:10.1109/TAES.2010.5595587

[6] Huang, J., Ma, G., and Liu, G., "Nonlinear Dynamics and Reconfiguration Control of Two-Satellite Coulomb Tether Formation at Libration Points," Aerospace Science and Technology, Vol. 39, Dec. 2014 , pp. 501-512. doi:10.1016/j.ast.2014.06.001

[7] Wang, S., and Schaub, H., "1-D Constrained Coulomb Structure Stabilization with Charge Saturation," AAS/AIAA Astrodynamics Specialists Conference, American Astronomical Soc. Paper 2007-267, Washington, D.C., Aug. 2007.

[8] Hussein, I. I., and Schaub, H., "Stability and Control of Relative Equilibria for the Three-Spacecraft Coulomb Tether Problem," Acta Astronautica, Vol. 65, Nos. 5-6, 2009, pp. 738-754. doi:10.1016/j.actaastro.2009.03.035

[9] Hogan, E. A., and Schaub, H., "Collinear Invariant Shapes for ThreeSpacecraft Coulomb Formations," Acta Astronautica, Vol. 72, MarchApril 2012, pp. 78-89. doi:10.1016/j.actaastro.2011.10.020

[10] Hogan, E. A., and Schaub, H., "Linear Stability and Shape Analysis of Spinning Three-Craft Coulomb Formations," Celestial Mechanics and Dynamical Astronomy, Vol. 112, No. 2, 2012, pp. 131-148. doi:10.1007/s10569-011-9387-6

[11] Jones, D. R., and Schaub, H., "Collinear Three-Craft Coulomb Formation Stability Analysis and Control," Journal of Guidance, Control, and Dynamics, Vol. 37, No. 1, 2013, pp. 224-232. doi: $10.2514 / 1.60293$

[12] Jasch, P. D., Hogan, E. A., and Schaub, H., "Out-of-Plane Stability Analysis of Collinear Spinning Three-Craft Coulomb Formations," Acta Astronautica, Vol. 88, July-Aug. 2013, pp. 89-97. doi:10.1016/j.actaastro.2013.03.005

[13] Saaj, C., Lappas, V., Schaub, H., and Izzo, D., "Hybrid Propulsion System for Formation Flying Using Electrostatic Forces," Aerospace Science and Technology, Vol. 14, No. 5, 2010, pp. 348-355. doi:10.1016/j.ast.2010.02.009

[14] Vasavada, H., and Schaub, H., "Analytic Solutions for Equal Mass FourCraft Static Coulomb Formation," Journal of the Astronautical Sciences, Vol. 56, No. 1, Jan.-March 2008, pp. 17-40. doi:10.1007/BF03256540

[15] Wang, S., and Schaub, H., "Coulomb Control of Nonequilibrium Fixed Shape Triangular Three-Vehicle Cluster," Journal of Guidance, Control, and Dynamics, Vol. 34, No. 1, Jan.-Feb. 2011, pp. 259-270. doi: $10.2514 / 1.47835$

[16] Wang, S., and Schaub, H., "Electrostatic Spacecraft Collision Avoidance Using Piece-Wise Constant Charges," Journal of Guidance, Control, and Dynamics, Vol. 33, No. 2, March-April 2010, pp. 510 520 . doi:10.2514/1.44397

[17] Schaub, H., Parker, G. G., and King, L. B., "Challenges and Prospect of Coulomb Formations," Journal of the Astronautical Sciences, Vol. 52, Jan.-June 2004, pp. 169-193. 